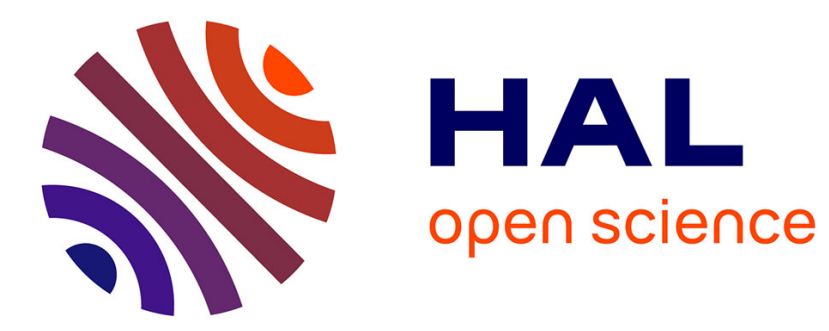

\title{
Suárez sur la causalité de l'agent et la notion de cause libre
}

\author{
Jean-Pascal Anfray
}

\section{To cite this version:}

Jean-Pascal Anfray. Suárez sur la causalité de l'agent et la notion de cause libre. Guillon, JeanBaptiste. Le libre arbitre, Collège de France, 2017, 10.4000/books.cdf.4944 . halshs-03183729

\section{HAL Id: halshs-03183729 \\ https://shs.hal.science/halshs-03183729}

Submitted on 28 Mar 2021

HAL is a multi-disciplinary open access archive for the deposit and dissemination of scientific research documents, whether they are published or not. The documents may come from teaching and research institutions in France or abroad, or from public or private research centers.
L'archive ouverte pluridisciplinaire HAL, est destinée au dépôt et à la diffusion de documents scientifiques de niveau recherche, publiés ou non, émanant des établissements d'enseignement et de recherche français ou étrangers, des laboratoires publics ou privés. 


\section{Suárez sur la causalité de l'agent et la notion de cause libre}

Jean-Pascal Anfray (ENS-PSL, Centre Mathesis, République des savoirs, USR 3608)

\section{Introduction}

Le problème du libre-arbitre comporte tout à la fois un aspect pratique et un aspect métaphysique. Dans sa dimension métaphysique, il engage notamment une conception de la causalité. Selon une conception incompatibiliste répandue, l'existence du libre arbitre suppose qu'un acte libre n'est pas causé par des événements antérieurs, mais directement par l'agent lui-même. Il s'agit de la thèse de la causalité de l'agent (agent causation). Le plus souvent, cette conception s'accompagne d'une métaphysique dualiste de la causalité, qui impose la distinction de deux régimes irréductibles de causalité : d'une part celui des événements naturels, comme la chute d'une pierre, l'éruption d'un volcan ou l'activation d'une protéine, qui obéissent à une causalité événementielle, dont les causes sont d'autres événements précédents les premiers; d'autre part, la causalité à l'œuvre dans les actions immédiates, comme la formation d'une intention ou une décision. Ces actions impliquent une forme exceptionnelle de causalité par un agent, irréductible à la causalité événementielle ${ }^{1}$. Ce qui distingue les actions libres ne réside pas seulement dans le caractère déterministe ou indéterministe de la causalité. La structure de la relation causale impliquée est elle-même fondamentalement différente. Dans le cas d'un effet naturel, un événement, cause l'occurrence d'un autre événement qui est l'effet dès lors qu'un ensemble de circonstances ou facteurs favorables sont posés. La causalité événementielle inscrit l'effet dans une chaîne causale, éventuellement indéterministe, comme la désintégration d'un atome de radium. La causalité à l'œuvre dans une action libre met au contraire en jeu un agent ou une personne et une action immédiate, un acte de volonté qui, dans la plupart des cas, déclenche un événement physique ultérieur. L'action immédiate est directement causée par l'agent qui est une substance et non un événement préalable. En causant une action, l'agent cause certes l'occurrence d'un événement, mais cet événement n'a pas d'autre cause en dehors de l'agent

\footnotetext{
${ }^{1}$ C'est le cas notamment de (Chisholm 1976a) et (Chisholm 1976, 53-88); (O’Connor 2000) ; (Clarke 2003); (Michon 2011, 67-71). Voir la discussion critique des théories de la causalité de l'agent dans (Pereboom 2014, chap.3).
} 
lui-même. Ainsi la causalité de l'agent n'implique pas de chaînes causales remontant dans le passé.

Mais il est possible d'envisager des théories dans lesquelles la causalité de l'agent n'est pas une forme de causalité régionale, mais s'inscrit dans une conception univoque de la causalité, comme impliquant fondamentalement des substances et leurs pouvoirs. Une telle conception peut être qualifiée de "néo-aristotélicienne » et a été récemment défendue par T. O’Connor et J.D. Jacobs. Selon ces derniers, toute causalité implique une causalité substantielle. Les substances sont les entités fondamentales qui possèdent des pouvoirs et sont les causes de différents effets en exerçant les pouvoirs correspondants. Dès lors, le critère de distinction entre les causes libres et celles qui ne le sont pas ne peut reposer sur la nature de la relation causale, mais repose sur le rôle des raisons de l'action et la nature spécifique de cette substance $^{2}$ :

Toute causalité est une causalité substantielle. Mais son caractère unique tient au type de substance, une substance consciente influencée par des raisons, et au type d'effet, une intention d'agir pour une certaine raison (Jacobs et O’Connor 2013, 186).

Cette conception néo-aristotélicienne de la causalité de l'agent fournit un point de comparaison pertinent avec la conception développée par Francisco Suárez (1548-1617) en particulier dans les Disputationes metaphysicae dont la première édition remonte à 1597 . Selon Suárez la liberté suppose la présence d'un pouvoir indéterminé de vouloir ou ne pas vouloir une certaine action. Ce pouvoir, identifié au libre arbitre, définit ce qu'est une cause libre. L'intérêt des analyses de Suárez ne réside pas tant dans sa caractérisation de la liberté d'indifférence, très courante à la fin du XVIe siècle et que l'on peut faire remonter à Duns Scot. Elle ne se trouve pas non plus uniquement dans son influence sur les débats ultérieurs sur le libre arbitre. Le principal intérêt de l'étude de la conception du jésuite espagnol tient à ce qu'il associe sa conception de ce qu'est une cause libre d'une part à une réflexion systématique sur la causalité, développée dans les disputes XII à XXVII des Disputationes metaphysicae, et d'autre part à une philosophie de l'esprit et de ses facultés extrêmement riche, principalement contenue dans son commentaire du De anima ${ }^{3}$. Nous nous intéresserons

\footnotetext{
2 (O’Connor et Jacobs 2013, 186) soutiennent qu'il s'agit d'une substance émergente.

${ }^{3}$ Pour le contexte historique, voir (Schmutz 2002) ; (Pink 2004); (Penner 2013). Près de vingt ans avant Suárez, Luis de Molina a défendu une conception similaire, qui provient à son tour de Duns Scot. Voir Molina, Concordia, disp.2 et (Anfray 2014). La formule quasi canonique positis omnibus requisitis ad agendum, velle potest vel non velle, se trouve déjà chez Ockham et les nominalistes du XIVe siècle. Voir Ockham, Quodlibeta septem, I, q.16, éd. J. C. Wey (Op. theol. IX, 87) : «voco libertatem potestatem qua possum indifferenter et contingenter diversa ponere, ita quod possum eumdem effectum causare vel non causare, nulla diversitate existente alibi extra istam potentiam ». Elle a des racines dans une tradition volontariste antérieure, remontant par-delà Duns Scot à Pierre Jean Olivi au XIIIe siècle. Voir (Putallaz 1995) et (Kent 1995).
} 
ici tout particulièrement aux implications de sa métaphysique de la causalité et de l'esprit sur sa conception de la liberté.

D'après Suárez, toute causalité met en jeu l'exercice de pouvoirs plus ou moins spécifiques par des agents. Ainsi, la causalité libre ne diffère pas structurellement de la causalité naturelle. En outre, l'expression «cause libre » (causa libera) est porteuse d'une certaine ambiguïté référentielle. Parfois en effet la cause libre désigne (1) l'être humain tout entier; parfois un composant de l'être humain, (2) son âme ; parfois enfin, (3) une faculté spécifique de l'être humain, sa volonté ou appétit rationnel. Au premier abord, cette ambiguïté peut apparaître comme bénigne et purement verbale. Le contexte permet souvent en effet de lever l'ambiguïté sémantique de l'expression «cause libre ». De plus, il est d'usage dans les reconstructions contemporaines des thèses scolastiques de paraphraser les énoncés de la forme «la volonté veut $X$ » en énoncés de la forme «l'agent $S$ veut $X$ » et de tenir cette paraphrase pour innocente ${ }^{4}$. Nous voudrions cependant montrer que cette paraphrase ne va pas nécessairement de soi chez un auteur tel que Suárez et que la polysémie de la cause libre soulève certains problèmes substantiels engageant l'interprétation de sa théorie de la liberté et pour lesquels il est nécessaire de se pencher sur l'étude de sa conception complexe de la causalité et des facultés de l'âme. Dans le cadre limité de cette étude, nous nous intéresserons en priorité aux actions immédiates ou actions de base, les volitions ou actus eliciti dans la terminologie scolastique, laissant de côté la question des actions libres impliquant des mouvements corporels, ou dans la terminologie scolastique les actus imperati. ${ }^{5} \mathrm{Ce}$ faisant, nous restreindrons notre propos à la question de savoir si la cause libre désigne l'âme ou une faculté particulière de l'âme, la volonté. L'âme qui est la forme substantielle de l'homme n'est une cause libre que parce qu'elle possède une faculté, la volonté. Or, Suárez admet que les facultés sont des entités réellement distinctes les unes des autres. Ce faisant, il prête le flanc à une critique bien connue à l'époque moderne à l'encontre des théories scolastiques des facultés, rendue célèbre par Locke, à savoir que la substantialisation des pouvoirs de l'âme conduit à une multiplication d'homoncules ou d'agents plus ou moins indépendants les uns

\footnotetext{
${ }^{4}$ Entre autres, Penner 2013, p.2, n.7 : «Je tiens pour acquis que selon Suárez les actions attribuées à la volonté ou à une autre faculté sont, d'abord et avant toutes choses, des actions de l'agent. Donc chaque fois qu'il dit que la volonté choisit quelque chose, nous devrions plutôt dire que l'agent choisit quelque chose ».

${ }^{5}$ Pour la distinction, voir Thomas d'Aquin, ST I-II, q.6, introd. ; q.8, introd. : « [actus] qui sunt immediate ipsius voluntatis, velut ab ipsa voluntate eliciti »; q.17, a.1-4. Les actus eliciti peuvent (1) concerner la fin. Il s'agit alors (1a) du vouloir (velle), (1b) de la jouissance (frui) ou (1c) de l'intention (intentio). Lorsqu'ils concernent les moyens, il s'agit (2a) du choix (electio) que précède la délibération (consilium); (2b) du consentement aux moyens (consensus) ou enfin (2c) de l'usage (uti).
} 
des autres ${ }^{6}$. Le réalisme des facultés réduirait ainsi l'âme à un réseau causal complexe quoique coordonné de pouvoirs ou facultés qui sont autant d'agents distincts, conception incompatible avec l'idée d'un agent unifié, essentielle à une théorie de la causalité de l'agent. La volonté, ainsi que les autres facultés, apparaissent comme autant de micro-agents qui ne permettent pas de rendre compte de la notion de contrôle requise pour la liberté et la responsabilité morale. L'idée de contrôle exige qu'une action libre soit susceptible d'une explication rationnelle et puisse être distinguée du résultat d'un processus aléatoire. Or la volonté, coupée de l'intellect, est dépourvue de raisons et motifs et ses actions apparaissent comme tout aussi indéterminées que des événements aléatoires ${ }^{7}$. De même, l'exercice du pouvoir des alternatives de la volonté, pouvoir de vouloir et de ne pas vouloir une certaine action, apparaît comme un pouvoir irrationnel d'agir contre ce que l'intellect propose comme un bien. Suárez donne lui-même de l'eau au moulin de ces objections :

Mais la volonté, parce qu'elle est pour ainsi dire aveugle, a besoin d'être gouvernée et dirigée par l'intellect et parce qu'elle tend vers son objet par une inclination volontaire, elle est capable de liberté. En cela, elle peut surpasser l'intellect, bien qu'absolument parlant elle lui soit inférieure en perfection ${ }^{8}$.

La volonté en tant que quasi-agent indépendant ne diffèrerait pas fondamentalement d'un mécanisme aléatoire et ne saurait disposer du type de contrôle requis pour le libre arbitre et la responsabilité. L'agent, entité indispensable à une conception incompatibiliste en termes de causalité de l'agent, tend ainsi à disparaître en se confondant avec un réseau complexe de pouvoirs causaux ${ }^{9}$. Nous essaierons d'étudier les ressources dont dispose Suárez pour répondre à ce défi. Nous présenterons d'abord les grands traits de la théorie du libre arbitre. Dans une deuxième partie, nous nous pencherons sur le rôle respectif des pouvoirs et de la substance dans la théorie de la causalité défendue par Suárez. Nous verrons ensuite de quelle façon cette théorie de la causalité fonctionne dans le cas de l'âme. Enfin nous étudierons en

\footnotetext{
${ }^{6}$ John Locke, Essay II, xxi, §20, 243-244: «But the fault has been, that faculties have been spoken of, and represented, as so many distinct agents. For it being asked, what it was that digested the meat in our stomachs? It was a ready, and very satisfactory answer to say, that it was the digestive faculty. What was it that made any thing out of the body? The expulsive faculty. What moved? The motive faculty. And so, in the mind, the intellectual faculty, or the understanding, understood; and the elective faculty, or the will, willed or commanded $»$.

${ }^{7}$ Voir en particulier l'argument du rollback de (Van Inwagen 2000) et le commentaire qu'en donne (Michon 2011, 107-122).

${ }^{8}$ DM XIX.5.25 : «Voluntas autem, quia est quasi caeca, indiget regula aut directione intellectus; quia vero tendit in suum obiectum per voluntariam inclinationem perfectam, capax est libertatis; et in hoc potest secundum quid excedere intellectum, quamvis simpliciter sit in perfectione inferior".

${ }^{9}$ (Pereboom 2014, chap.2) nomme argument de l'agent évanescent (disappearing agent) 1'objection selon laquelle l'agent dans une conception libertarienne événementielle ne dispose pas d'un contrôle causal suffisant pour fonder la responsabilité morale. Mais dès lors qu'une conception de la causalité ne permet pas de penser un agent unifié, elle offre prise à une objection similaire.
} 
détail la réponse de Suárez à deux problèmes centraux pour toute théorie libertarienne : les problèmes apparentés de la rationalité et du contrôle causal.

\title{
1. Esquisse de la théorie de la causalité libre et du libre arbitre de Suárez
}

Dans la dix-neuvième des Disputes métaphysiques, Suárez aborde l'existence et la nature des causes libres, qui sont une modalité des causes efficientes.

L'existence de la liberté est un fait indubitable, attesté dans l'expérience interne de nos propres décisions :

\begin{abstract}
En second lieu, nous pouvons argumenter à partir de l'expérience. Nous sentons avec évidence en effet qu'il est en notre pouvoir de faire quelque chose ou de l'omettre et à cette fin nous employons la raison et le discours, ainsi que la délibération, afin d'être inclinés à un parti plutôt qu'à un autre. Le choix réside donc dans notre libre-arbitre. Autrement, comme le dit à juste titre Damascène, ce serait en vain que nous aurait été donné ce pouvoir de soupeser et de délibérer. À cela se rattache également cette manière courante d'opérer et de gouverner les actions humaines par des conseils, par des lois et des préceptes, par des exhortations et des réprimandes, par la promesse de récompenses et l'infliction de peines, toutes choses qui seraient absolument vaines si l'homme agissait par la nécessité de la nature et non par sa liberté ${ }^{10}$.
\end{abstract}

L'expérience immédiate d'un pouvoir des contraires fournit ainsi un argument direct en faveur de l'existence de la liberté. La suite du texte présente un second argument, par les conséquences : pour donner sens à la louange et blâme, aux récompenses et châtiments, conseils et menaces, il faut que nous soyons libres ${ }^{11}$. Autrement dit nos attitudes et pratiques réactives, pour reprendre la terminologie de Strawson, requièrent l'existence d'une liberté incompatible avec la détermination par des causes antécédentes ${ }^{12}$.

Les causes libres se distinguent des causes naturelles. Ces dernières sont déterminées à produire des effets d'un certain type dans des circonstances données. Les agents dépourvus d'usage de la raison sont des causes naturelles. Réciproquement, tous les agents rationnels et seulement eux sont des causes libres. La rationalité est ainsi une première condition de la liberté. La liberté suppose en outre un pouvoir actif capable de produire des actions contraires.

\footnotetext{
${ }^{10}$ DM XIX.2.13 : «Secundo argumentari possumus ab experientia; experimur enim evidenter situm esse in nostra potestate aliquid agere vel omittere, et ad hoc utimur ratione et discursu ac consultatione ut in unam partem potius quam in aliam inclinemur; est ergo electio posita in nostro arbitrio; alioqui, ut recte dixit Damascenus supra, fuisset nobis data superflue haec deliberandi et consultandi vis. Huc accedit usitatus modus operandi et gubernandi humanas actiones per consilia, per leges et praecepta, per exhortationes ac reprehensiones, per promissiones praemiorum et comminiones poenarum, quae omnia supervacanea essent si homo necessitate naturae et non libertate sua operaretur $»$.

${ }^{11}$ Suárez considère l'objection selon laquelle l'expérience établit seulement le rôle des attitudes réactives dans nos actions, mais non sa conception incompatibiliste de la liberté. Il répond à cette objection en affirmant que nous avons l'expérience, face à la promesse d'une récompense ou à la menace d'un châtiment, du pouvoir d'agir à l'encontre. Cf. DM XIX.2.15 : « experimur etiam post cognitam comminationem poenae aut promissionem praemii situm esse in potestate nostra ea ratione moveri aut non moveri ».

${ }^{12} C f$. (Strawson 1962).
} 
Une cause libre opère en effet de manière contingente et implique un pouvoir des contraires. Une cause libre $S$ est telle que même si tous les réquisits d'une certaine action $A$ sont donnés, S peut accomplir $A$ ou s'abstenir d'accomplir $A$ :

Il y a dans l'homme une faculté active qui, par soi et par sa nature intrinsèque et particulière, n'est pas déterminée à une action seulement, mais est de soi indifférente à agir ainsi ou autrement et à agir et ne pas agir, tous les réquisits de l'action étant posés ${ }^{13}$.

Ce pouvoir des alternatives n'est pas un simple pouvoir conditionnel ou in sensu diviso - le pouvoir de ne pas accomplir $A$ si les circonstances dans lesquelles se trouve $S$ avaient été différentes. La clause positis omnibus requisitis signifie qu'il s'agit d'un pouvoir absolu des contraires, ou in sensu composito - le pouvoir d'accomplir $A$ ou non- $A$, les circonstances actuelles antérieurement requises à l'exercice de la volonté restant fixes ${ }^{14}$.

Les conditions de la liberté (rationalité, activité, pouvoir des contraires) impliquent en outre la présence de plusieurs facultés distinctes dans l'âme, l'intellect et la volonté. Il s'agit de deux facultés rationnelles de l'âme, qui est l'unique forme substantielle de l'homme. Un débat central pour la philosophie de l'esprit médiévale depuis le début du XIIIe siècle concerne en effet l'unité ou la pluralité des formes substantielles. Depuis Avicenne, un certain nombre de penseurs soutiennent qu'une substance telle que l'homme possède une pluralité de formes substantielles, chacune chargées de rendre compte d'un ensemble de capacités. Ockham est un exemple typique. Il distingue en effet une forme de la corporéité, une forme sensorielle chargée des fonctions vitales (nutritives et sensori-motrices) et une forme intellectuelle (qui accomplit les fonctions cognitives et conatives supérieures) ${ }^{15}$. Au contraire, prenant le parti de Thomas d'Aquin, Suárez soutient l'unité de l'âme, tout en maintenant que celle-ci contient un ensemble de facultés distinctes qui forment une hiérarchie : les facultés nutritives, sensitives et rationnelle ${ }^{16}$. Les premières facultés supposent des organes physiologiques adaptés. Les facultés rationnelles sont au contraire dépourvues d'organes spécifiques.

\footnotetext{
${ }^{13}$ DM XIX.2.11: « esse in homine facultatem aliquam activam quae ex se et ex sua intrinseca ac particulari natura non est determinata ad unum tantum, sed indifferens ex se ad hoc vel illud operandum, et ad operandum et non operandum, positis omnibus requisitis ad agendum ». Voir aussi le titre de la section IV : " causam liberam esse quae, positis omnibus requisitis ad agendum, potest agere et non agere ».

${ }^{14}$ DM XIX.4.11: «Ut ergo facultas liberum usum habeat, necesse est ut, positis omnibus conditionibus praerequisitis antecedenter seu in actu primo, ex interna vi et facultate sua possit agere et non agere ». Suárez exclut ainsi les réquisits concomitants : étant donné la volonté de faire $A$, l'agent n'a pas le pouvoir de vouloir non- $A$. Mais cette volonté est un réquisit concomitant. De même, le concours divin à l'action fait partie des réquisits concomitants et non une détermination antécédente ou motio praevia. Cette dernière abolit le pouvoir des contraires et ainsi est incompatible avec la liberté. Sur la terminologie du sens composé et divisé, cf. DM XIX.4.16 et (Schmutz 2002).

${ }^{15}$ Ockham, Quodlibeta II, q. 10-11, Op. theol. IX, 156-164. Sur l'histoire de la controverse entre pluralistes et unitariens, voir (Zavalloni 1951) et (Pasnau 2011, 574-605).

${ }^{16}$ Thomas d'Aquin, ST I, q.76, a.3, in corp.; Suárez, DM XV.10.
} 
Dans la mesure où la liberté présuppose la rationalité, elle ne peut résider que dans une âme pourvue de facultés rationnelles. Il revient en effet à l'intellect de connaître le vrai et le bien. C'est dans la mesure où l'intellect représente un objet sous l'aspect d'un bien que l'agent libre est sensible aux raisons. Mais c'est par la volonté que l'âme est libre. Celle-ci est définie à la fois comme un pouvoir actif et un pouvoir des contraires :

Il y a dans l'homme une puissance active, libre en vertu de sa propre force et nature intrinsèque, c'est-àdire, qui possède une telle souveraineté sur son action, qu'il est en son pouvoir de l'exercer et de ne pas l'exercer, et en conséquence de choisir une action ou l'action opposée ${ }^{17}$.

Suárez affirme que la volonté est le pouvoir dans lequel réside formellement la liberté. Il identifie ainsi volonté, faculté libre et liberum arbitrium. Ce faisant, il prend parti contre la tradition thomiste qui fait de l'intellect la racine de la liberté au sens où l'indétermination de la volonté provient de l'indétermination des raisons présentes dans l'intellect ${ }^{18}$. L'indifférence de la volonté dépend selon les thomistes de l'indifférence du jugement pratique au sujet d'une action particulière. Ces jugements ne sont indifférents que lorsqu'ils portent sur des biens particuliers qui sont imparfaits en ce qu'ils sont bons sous un aspect, mais pas sous tous les aspects. Ainsi un repas gastronomique est un plaisir gustatif et un bien sous cet aspect, mais il peut être mauvais pour un régime ou pour la santé, et sous cet aspect, ce n'est pas un bien. La multiplicité des biens finis est la condition sous laquelle la volonté possède le pouvoir de vouloir une action ou de s'en abstenir. Mais si l'intellect représente un bien absolu, il n'y a plus alors d'indifférence dans la volonté qui est nécessitée à vouloir ce bien tant qu'il lui est présenté par l'intellect ${ }^{19}$.

Selon Suárez, la volonté présuppose effectivement un jugement de l'intellect sur un bien à poursuivre et ne peut pas agir sans la représentation d'un objet comme un tel bien. Elle est donc une faculté essentiellement sensible aux raisons. Cependant ces raisons ne suffisent pas à déterminer le choix de la volonté. L'indifférence en tant qu'elle s'attache aux biens présentés par l'intellect peut être appelée une indifférence objective. La thèse de Suárez est

\footnotetext{
${ }^{17}$ DM XIX.2.18 : «esse in homine aliquam potentiam activam ex sua vi et intrinseca natura liberam, id est, habentem tale dominium suae actionis ut in eius potestate sit eam exercere et non exercere, et consequenter unam vel aliam seu oppositam actionem elicere ».

${ }^{18}$ Thomas d'Aquin, ST I-II, 17, a.1, ad 2: «Ad secundum dicendum quod radix libertatis est voluntas sicut subiectum, sed sicut causa, est ratio. Ex hoc enim voluntas libere potest ad diversa ferri, quia ratio potest habere diversas conceptiones boni. Et ideo philosophi definiunt liberum arbitrium quod est liberum de ratione iudicium, quasi ratio sit causa libertatis $»$.

${ }^{19}$ Thomas d'Aquin, ST I, 82, 2, ad 2 : «Ad primum ergo dicendum quod voluntas in nihil potest tendere nisi sub ratione boni. Sed quia bonum est multiplex, propter hoc non ex necessitate determinatur ad unum »; ST I, 83, 1 : «Sed quia iudicium istud non est ex naturali instinctu in particulari operabili, sed ex collatione quadam rationis; ideo agit libero iudicio, potens in diversa ferri. Ratio enim circa contingentia habet viam ad opposita; ut patet in dialecticis syllogismis, et rhetoricis persuasionibus. Particularia autem operabilia sunt quaedam contingentia, et ideo circa ea iudicium rationis ad diversa se habet, et non est determinatum ad unum. Et pro tanto necesse est quod homo sit liberi arbitrii, ex hoc ipso quod rationalis est $»$.
} 
alors que l'indifférence objective n'est pas une condition suffisante pour définir une conception robuste de la liberté qui requiert une indifférence de pouvoir (indifferentia potentiae) autrement dit un pouvoir indéterminé des contraires.

Ce pouvoir des contraires dote l'agent de trois sortes d'indifférence. Il y a ainsi trois types de manifestations possibles de ce pouvoir. Les agents libres peuvent en effet être indifférents par rapport à l'exercice de la volonté. La volonté peut en effet ou bien vouloir une action, par exemple lire un livre, ou s'en abstenir, ne pas lire un livre. Elle peut également être indifférente à l'égard de la spécification de l'objet de l'action. Ainsi je peux lire un roman ou bien un essai. Enfin, il y a une indifférence dans la spécification de l'attitude à l'égard de l'objet (je peux aimer ce roman ou le haïr) ${ }^{20}$. La première forme d'indifférence est essentielle à la volonté. En dehors du cas exceptionnel de la vision béatifique, elle est toujours présente. Ainsi, en cette vie, même si l'objet proposé à la volonté est la finis ultimus ou Dieu, nous jouissons d'une liberté d'exercice par laquelle nous pouvons ne pas adhérer à cette fin. En dehors de ce cas, l'indifférence d'exercice s'accompagne cependant d'une liberté de spécification. Autrement dit, le pouvoir de refuser une action présentée par l'intellect comme un bien implique, dans les cas normaux, un pouvoir de vouloir une autre action représentée comme un bien ${ }^{21}$. Nous reviendrons sur la manière dont Suárez défend cette conception de la liberté contre les deux objections classiques de la rationalité et du contrôle dans la dernière partie de l'article. Auparavant, il convient de relier cette conception de la liberté à la conception suarézienne de la cause efficiente en générale et aux rapports de causalité entre les différentes facultés au sein de l'âme humaine.

\section{Causalité, substances et pouvoirs}

Les causes libres comme les causes nécessaires relèvent de la causalité efficiente qui est la forme fondamentale de la causalité ${ }^{22}$. La cause efficiente est définie comme « un principe extrinsèque par soi par lequel un changement existe en premier lieu $»^{23}$. Il s'agit d'un principe extrinsèque par contraste avec la cause formelle et la cause matérielle qui sont des principes constitutifs des choses, qui n'existent pas indépendamment de cette chose. En outre la cause efficiente est un principe de changement a quo, par quoi elle diffère de la cause finale (DM

\footnotetext{
${ }^{20}$ DM XIX.4.9 ; ce point est commenté dans (Schmid 2015, 89).

${ }^{21}$ DM XIX.4.10 : «Cum ergo dicitur liberum esse quod, positis omnibus requisitis ad agendum, potest agere et non agere, intelligendum est de praerequisitis antecedenter et in actu primo, non de aliis ».

${ }^{22}$ Cf. DM XII.2.3 : « causa est principium per se influens esse in aliud ». Comme l'indique Suárez, c'est la cause efficiente qui est la principale responsable de l'existence, d'où découle la primauté de l'efficience ; cf. DM XXVII.1.10 : « nam efficiens propriissime influit esse ». Sur la causalité efficiente chez Suárez, voir (Schmid 2015); (Carraud 2002); (Schmaltz 2008).

${ }^{23}$ DM XVII.1.2 : « Dici posset causa efficiens esse principium per se, extrinsecum, a quo primo est mutatio ».
} 
XVII.1.3). Il est essentiel pour comprendre la position de Suárez de distinguer la cause, l'effet et l'exercice de la causalité. Cette distinction permet de comprendre comment la cause efficiente peut être identifiée à une substance dont l'action particulière constitue la causalité débouchant sur un effet. La causalité désigne l'action de l'agent. Il ne s'agit pas d'une propriété de l'agent ou de la cause efficiente, mais d'un mode de l'effet produit. Dans l'ontologie suarézienne, un mode n'est pas une chose distincte, contrairement à une substance ou à un accident tel que la quantité ou la qualité. Un mode désigne une manière d'être d'une chose, qui affecte celle-ci et ne peut exister sans elle, mais qui n'ajoute pas une entité propre ${ }^{24}$. En posant l'action comme un mode de l'effet et non de la cause, Suárez indique que le critère d'identité de l'action est donné par son résultat ou son effet. Ainsi l'action de découper une feuille de papier est définie par son résultat, le fait que la feuille soit découpée et bien que cette action dépende d'une cause extérieure, son identité dépend de l'effet précis : telle action de couper diffère de telle autre action non en elle-même, ni par l'agent, mais en vertu de la nature de l' effet $^{25}$.

Comme nous l'avons déjà vu, les causes efficientes ne sont pas des événements mais des choses. Cela ne signifie pas pour autant qu'une cause efficiente agisse automatiquement du simple fait de son existence. L'exercice de la causalité d'une cause efficiente dépend de certaines circonstances qui sont les réquisits de l'action. Dans le cas des causes naturelles ou nécessaires, Suárez définit une série de tels réquisits (DM XIX.1.2-4). Tout d'abord, l'agent requiert un patient sur lequel l'effet peut se produire. Ce patient doit en outre être suffisamment proche et pourvu d'un pouvoir passif corrélé au pouvoir actif de la cause. De plus, il ne doit pas y avoir de causes empêchant l'activation de ce pouvoir. Enfin, les causes n'agissent pas d'elles-mêmes, mais ont besoin du concours de la cause première pour agir. Chacun de ces réquisits constitue une condition nécessaire à l'exercice d'une cause efficiente. Lorsque tous ces réquisits sont posés avec une cause naturelle, celle-ci agit nécessairement. Cependant il s'agit bien de réquisits pour l'action des causes et non de causes, même partielles.

\footnotetext{
${ }^{24}$ DM VII.1.16-24. Sur la théorie suarézienne des modes, voir (Menn 1997) et (Pasnau 2011, chap.13).

${ }^{25}$ (Schmid 2015, 98-103) rattache la thèse selon laquelle l'action est un mode de l'effet à deux autres raisons. La première est la conception aristotélicienne du mouvement comme actualisation de ce qui est en puissance en tant qu'il est en puissance (Physique III, 3, 202a13-b29). Dans cette conception, l'agent ne change pas, mais seulement le patient. L'autre raison est de nature théologique : Dieu est la cause efficiente du monde. Mais Dieu est acte pur et immuable et l'action créatrice ne peut donc introduire un changement en lui, mais seulement dans l'effet. Comme le remarque Schmid, cela soulève le problème de l'attribution d'un sujet de l'action dans le cas de la création ex nihilo. La solution consiste à dire que l'action n'est pas inhérente à l'effet comme à un sujet, mais l'accompagne (adhaerens) en étant à la fois productrice de l'existence de cette chose et ontologiquement dépendante de celle-ci (DM XLVIII.4.13).
} 
Sur le plan ontologique, l'analyse de Suárez mobilise ainsi deux catégories distinctes : des substances et des pouvoirs. Les causes efficientes sont des substances pourvues d'un pouvoir dont la manifestation est l'effet produit par l'agent. Cet effet se produit dans un patient luimême pourvu d'un pouvoir passif corrélé au pouvoir actif, pouvoir susceptible de recevoir l'action de la cause active. Ainsi, la cause efficiente peut désigner aussi bien la substance ellemême, ou sa forme substantielle, qu'un pouvoir actif de cette substance. En toute rigueur cependant, il convient de les distinguer. Suárez désigne en effet l'agent en tant que substance comme un «principe en tant que ce qui » (principium ut quod) ou principe-quod. Le pouvoir ou la faculté est au contraire désigné comme un «principe en vertu de quoi » (principium ut $q u o)$ ou principe-quo :

En effet cette cause principale [...] [est double]: l'une est celle qui opère, l'autre est le principe principal de l'opération, ce qu'on appelle ordinairement la cause principale ut quod et ut quo [...]. L'effet quant à l'efficience pure peut être attribuée véritablement et proprement à la cause principale opérante $u$ quod. $^{26}$

Il est manifeste que selon le premier mode [ut quod] c'est le suppôt qui opère. Mais si on le considère formellement, en tant que cause par soi d'une action accidentelle, il doit être pris en tant qu'il est le sujet d'une forme accidentelle qui est le principe d'une telle action. C'est ainsi qu'Aristote disait que Polyclète fabrique une statue par accident, alors que l'artisan la fabrique par soi. De cette façon donc, on dit l'accident cause principale en tant que principe quo, tandis que le suppôt en tant qu'il est le sujet d'un accident, en tant que principe $q u o d^{27}$.

L'exemple précédent permet d'illustrer cette distinction : le savoir faire d'un artiste est la cause principale $u t$ quo de la sculpture, mais la cause principale ut quod est l'artiste lui-même ou l'agent. Mais pour produire cette sculpture, l'artiste doit disposer du savoir-faire suffisant. Plus généralement, le suppositum est la cause efficiente d'un effet particulier non en tant que sujet nu, dépourvu de pouvoirs, mais seulement en tant qu'il est spécifié par de tels pouvoirs.

Les choses paraissent simples : les substances ou supposita sont les sources de l'activité, mais celle-ci est spécifiée par une série de pouvoirs qui correspondent aux propriétés essentielles et accidentelles de cet agent. Réciproquement, les pouvoirs n'agissent pas au sens où ils ne sont pas les agents du changement, mais ce par quoi les agents agissent.

Toutefois, les choses sont en réalité plus complexes qu'il ne semble. En effet, Suárez propose parfois une analyse de la causalité dans laquelle non seulement les substances, mais

\footnotetext{
${ }^{26}$ DM XVII.2.7 : « Haec enim causa principalis, ut ratio facta declarat, alia est quae operatur, alia quae est principium principale operandi, quae solent dici causa principalis ut quod et ut quo (...) Effectus quoad puram efficientiam omni principali causae operanti ut quod vere ac proprie attribui possit ».

${ }^{27}$ DM XVIII.6.2 : «Constat enim priori modo [i.e. ut quod] suppositum esse quod operatur; si autem sumatur formaliter, ut est per se causa accidentalis actionis, accipi debet ut substat formae accidentali quae est principium talis actionis; sic enim dixit Aristoteles Polycletum per accidens efficere statuam, artificem autem per se. Hoc ergo modo dicitur accidens causa principalis ut principium quo, suppositum vero ut substans accidenti, ut principium quod».
} 
également les pouvoirs sont des causes, comprises comme principes-quod. Ces pouvoirs sont des propriétés ou des accidents réels dans les choses. Par exemple la chaleur est une forme qui peut exister dans plusieurs sujets différents et qui possède en même temps un pouvoir, le pouvoir de chauffer, lequel peut également se manifester dans des sujets différents. Ainsi la chaleur qui existe dans le feu produit la chaleur dans l'eau contenue dans la casserole. Or l'eau chaude réchauffe également l'air environnant. Ce n'est pas la substance de l'eau qui réchauffe l'air, mais seulement le pouvoir accidentel et temporaire qu'elle a acquis. En un sens, il semble que le pouvoir de la chaleur cause directement par elle-même la manifestation de son effet $^{28}$. La théologie offre le cas le plus net d'une telle contribution causale directe des pouvoirs. Ainsi dans l'Eucharistie, la substance du pain disparait, remplacée par le corps du Christ et seules ses espèces subsistent. Néanmoins les pouvoirs naturels du pain n'ont pas disparu pour autant : l'hostie consacrée possède ainsi le pouvoir de nourrir ${ }^{29}$. Or la quantité dans laquelle inhèrent miraculeusement les accidents du pain ne constitue pas une source d'activité. Par conséquent, le pouvoir nutritif contenu dans les espèces du pain est à la fois un principe $u t$ quod et $u t$ quo, autrement dit il est pleinement la cause efficiente de l'effet.

Comme souvent dans la métaphysique de Suárez, ce cas exceptionnel fait apparaître une structure qui peut être généralisée. La généralisation du cas eucharistique, fait apparaît les pouvoirs comme d'authentiques agents causaux. Il semble avoir pour résultat de réduire les substances au rang de simples substrats de ces pouvoirs, conformément à la critique de Locke.

Cette conséquence, la quasi-autonomie des pouvoirs causaux, ne vaut toutefois que pour un certain type de pouvoirs, les pouvoirs des êtres naturels dépourvus de vie. Les êtres vivants au contraire possèdent des pouvoirs et facultés produisant des actions vitales qu'elles sont incapables de produire indépendamment de la forme substantielle à laquelle elles sont attachées $^{30}$. Suárez justifie cette affirmation en considérant un cas faisant intervenir la considération de la toute-puissance divine dans son Commentaire sur le De anima. Supposons que Dieu crée une âme et empêche aussitôt l'émanation naturelle des facultés de l'âme, en particulier de l'intellect. De la possibilité d'existence de l'âme sans ses facultés découle la distinction réelle entre l'âme et ces facultés. Prolongeant la tradition thomiste, Suárez soutient en effet que les facultés émanent de l'essence de l'âme, sans toutefois faire partie de cette

\footnotetext{
${ }^{28} C f$. (Schmid 2015, 105-108).

${ }^{29}$ CDA III.1.21 : " Dicit esse certum secundum fidem passionem esse separabilem a substantia, quia in sacramento Eucharistiae manet passio panis: nutrit enim. Probatur opinio, quia nulla est implicatio contradictionis in ea re. Sunt enim entitates distinctae, et non habent essentialem dependentiam ».

${ }^{30}$ Suárez recourt fréquemment à la notion d'action vitale. Cette notion est assez rare dans la scolastique médiévale. La caractérisation des actes d'un être vivant comme des actions vitales apparaît néanmoins chez un auteur comme Olivi. Cf. Pierre Jean Olivi, Quaestiones in secundum librum Sententiarum, q.71 (II, 644).
} 
essence $^{31}$. Il ajoute que ces différentes facultés possèdent chacune une nature spécifique : l'intellect pense, la volonté veut, la faculté nutritive est chargée du métabolisme de l'organisme, etc. Chacune de ces natures est une res distincte produisant des effets distincts. Suárez comprend l'émanation des facultés à partir de l'âme sur le modèle d'une causalité efficiente continuellement renouvelée ${ }^{32}$. Ainsi, l'hypothèse de l'intervention divine permet de conclure que l'âme peut exister sans ses facultés. Privée d'intellect, l'âme continuerait à exister, mais serait incapable de penser. La réciproque n'est cependant pas vraie :

Mais de nouveau l'on demande si l'intellect séparé peut penser, de même que la chaleur peut chauffer. On répond que cela n'est pas possible, parce que la pensée (intellectio) est une action vitale qui pour cette raison dépend essentiellement d'un premier principe de vie, sans lequel elle ne peut exister ${ }^{33}$.

Suárez concède effectivement qu'un intellect pourrait subsister séparé de la substance de l'âme ${ }^{34}$. Il serait cependant incapable de produire son activité propre, la pensée. La faculté vitale requiert en effet essentiellement le sujet pour produire son activité. Plus précisément, l'opération d'une faculté dépend essentiellement de la forme substantielle qui, pour recourir à une métaphore, fournit l'énergie causale sans laquelle ce pouvoir ne pourrait agir. Il existe ainsi une différence fondamentale entre les substances naturelles vivantes et les autres. Ces dernières peuvent être comprises comme de simples substrats de réseaux causaux complexes de différents pouvoirs. Les pouvoirs des êtres vivants possèdent une étroite intégration. L'activité des pouvoirs vitaux dépend essentiellement de leur forme substantielle unique et ces pouvoirs vitaux ne sont donc pas de petits homoncules autonomes.

Pour cette raison, lorsqu'il identifie les principes d'une action libre, Suárez affirme que le principe principal ut quod est l'homme et que le principe principal ut quo n'est pas la volonté, mais l'âme rationnelle qui est la forme substantielle de l'homme : « le principe principal quo de l'action libre doit toujours être une substance ou une forme substantielle spirituelle ${ }^{35}$. La

\footnotetext{
${ }^{31}$ Thomas d'Aquin, ST I, q.77, a.6, ad 1. Voir (Perler 2015, 108-110).

${ }^{32}$ CDA III.3.1 et 3 .

${ }^{33}$ CDA III.1.22 : «Sed quaeres rursus, utrum intellectus separatus possit intelligere, sicut calor calefacere. Respondetur: Non posse, quia intellectio est actio vitalis, et ideo intrinsece pendet a primo principio vitae, sine quo esse non potest $\gg$.

${ }^{34}$ Certains commentateurs nient que le fait que les facultés soient des res distinctes implique la possibilité de leur existence séparée indépendamment de l'âme. Les facultés selon (Shields 2014, 207) ont une présence virtuelle dans l'âme. On peut concéder que les facultés ne peuvent pas être créées indépendamment de l'âme. Mais le passage précédent indique clairement la possibilité d'une conservation indépendante de l'âme et donc une forme de séparabilité.

${ }^{35}$ DM XIX.5.2 : «principium principale quo liberae actionis semper esse substantiam aliquam seu substantialem formam spiritualem ». Le contexte immédiat de ce passage est une réfutation de la possibilité d'attribuer la liberté à une substance matérielle, du fait que seule une substance immatérielle agit avec intelligence.
} 
volonté n'est ainsi qu'un principe proche $u t$ quo de l'action libre ${ }^{36}$. Au sens strict, ce n'est pas la volonté qui agit et cause la volition, mais l'âme rationnelle. Les pouvoirs vitaux s'apparentent ainsi plutôt à de simples instruments de la forme substantielle, qui est l'agent causal au sens strict. Suárez atténue cependant la portée de cette comparaison en ajoutant que les facultés et pouvoirs mentaux peuvent être considérés comme des "principes principaux » de l'action, à condition de les rapporter chacun à leur ordre propre. La comparaison pertinente est alors celle d'un principe universel et d'un principe "prochain ». L'âme fournit une forme de pouvoir causal indéterminé que les différentes facultés viennent spécifier ${ }^{37}$.

\section{La causalité dans l'âme : pouvoirs vitaux et la sympathie des facultés de l'âme.}

L'âme est donc la cause principale de l'activité et ce par quoi les facultés sont activées. D'un autre côté chaque faculté est responsable d'un type d'activité bien défini et de lui seul. La question qui se pose est alors de savoir comment s'articulent les activités des différentes facultés de l'âme. Les pouvoirs vitaux ont une particularité : ils produisent d'abord des actions immanentes, même lorsqu'ils s'accompagnent d'actions transitives. Une action est transitive lorsque l'effet existe en dehors de l'agent. Construire une maison ou marcher sont des paradigmes d'actions transitives, car les effets de ces actions sont corporels et existent en dehors de l'âme de l'architecte ou de l'animal qui marche. Une action immanente est à l'inverse une action dont l'effet immédiat réside dans le pouvoir causal lui-même. L'intellection et la volition sont les paradigmes d'actions immanentes ${ }^{38}$.

Selon Suárez donc, les actes produits par les facultés vitales ont d'abord pour terme la faculté elle-même. Mais le point crucial est qu'en retour les activités des pouvoirs vitaux ne sont pas à leur tour les effets transitifs de l'activité d'autres pouvoirs : «l'action d'un pouvoir vital ne produit pas l'action vitale d'un autre pouvoir $»^{39}$. Comme le souligne S. Knuuttila, «les pouvoirs vitaux sont immunisés aux influences causales extérieures ${ }^{40}$. Cette thèse est

\footnotetext{
${ }^{36}$ DM XIX.5.3 : «principium proximum actionis liberae esse facultatem spiritualem». Cela vaut des actes immédiats ou actus eliciti. Dans le cas des actus imperati que sont les actions impliquant des mouvements corporels, la dénomination de libre provient de leur dépendance à l'égard de cette faculté immatérielle.

${ }^{37}$ DM XVIII.6.3 : «potentiae animae ad actiones vitales merito dici possunt instrumenta coniuncta animae, quatenus illi subordinantur sicut principali formae, ut sine actuali influxu et quasi motione illius suas operationes efficere non possint, quamvis alia ratione possint dici principia principalia, saltem partialia, quia, scilicet, virtus quam in se habent et qua cooperantur ad effectum est ex se proportionata et commensurata tali effectui, quia non excedit perfectionem ipsius facultatis; loquor enim de actionibus connaturalibus. Unde etiam possunt potentia et forma comparari ut principium universale et proximum, utrumque vero principale et sufficiens ac totale in suo ordine; tamen, quia inter illa duo principalius est forma, cui subordinatur potentia, ideo haec subordinatio et dependentia nomine instrumenti significari potest $»$.

${ }^{38}$ Pour la distinction, voir DM XLVIII.2.1. Suárez discute plus en détail cette distinction dans DM XVIII.7.4551.

${ }^{39}$ CDA XII.3.9 : « actus unius potentiae vitalis non efficit actum alterius potentiae ».

${ }^{40} C f$. (Knuuttila 2014,196).
} 
ancrée dans l'idée que l'âme est un principe de vie et en ce sens la source des activités des êtres vivants. Cela implique non seulement que les actes vitaux ne sont pas les effets de causes efficientes externes, mais encore l'absence de causalité efficiente entre les actions vitales issues de pouvoirs distincts au sein d'une même substance ${ }^{41}$. Cela n'empêche pas cependant la détermination des actions vitales par des réquisits extérieurs ou par les actions d'autres pouvoirs d'une même substance. Suárez recourt alors à l'idée qu'un pouvoir vital est « excité » ou « appliqué » à son action propre :

DM XXII.2.28. L'excitation des puissances de l'âme se fait de deux manières. La première est par l'objet, ou bien dans le genre de la cause efficiente, comme dans les puissances cognitives, ou bien dans le genre de la fin, comme dans les puissances appétitives [...]. L'excitation relative aux puissances de l'âme se produit d'une autre manière, par une efficience quasi morale, selon une sympathie naturelle qu'elles possèdent, du fait qu'elles sont enracinées dans la même essence de l'âme, avec une subordination entre elles. On l'appelle aussi d'ordinaire motion ou application d'une puissance par une autre, à la manière dont la puissance motrice existant dans les muscles est excitée ou appliquée par l'appétit, et de cette façon on dit que la volonté meut toutes les puissances de l'âme ${ }^{42}$.

$\mathrm{Au}$ premier abord, toutes les connexions non-causales peuvent être assimilées à des facteurs déclenchants de l'activité des pouvoirs. Le fait d' "appliquer" une faculté à son opération renvoie à une connexion non-causale entre une certaine condition et l'activité de cette faculté. Suárez recourt à cette notion pour caractériser aussi bien la relation entre un réquisit extérieur et la faculté que la relation entre les différentes facultés d'une même âme. Néanmoins ces deux relations sont dissemblables. En effet, dans la relation entre un réquisit externe et une faculté, par exemple entre une sensation et un objet sensible, celui-ci est, avec le medium de la sensation, une simple condition nécessaire déterminant la faculté sensitive à produire une sensation donnée (CDA V.4.4). Il s'agit alors d'une corrélation non-causale mais nécessaire entre une condition et l'activation d'une faculté. Cette corrélation est à son tour fondée dans l'ordre général de la nature instauré par Dieu et ne demande pas un fondement ultérieur dans la nature intrinsèque du pouvoir sensible.

Les choses diffèrent lorsqu'il s'agit de la relation entre les activités de puissances vitales distinctes. Dans ce cas en effet, Suárez caractérise cette coordination entre les facultés de l'âme en parlant d'une harmonie, d'une concorde, d'un consensus (consensio) ou encore

\footnotetext{
${ }^{41}$ CDA V.3.3 : «de ratione actionis vitalis est quod fiat a principio intrinseco activo »; V.4.4.

${ }^{42}$ DM XXII.2.28 : «Excitatio autem potentiarum animae duobus modis fit. Uno, ab obiecto, vel in genere efficientis, ut in potentiis cognoscitivis, vel in genere finis, ut in appetitivis [...]. Alio modo fit excitatio circa potentias animae per quamdam efficientiam quasi moralem, per naturalem sympathiam quam habent, ex eo quod in eadem essentia animae radicantur, cum subordinatione inter se, quae etiam dici solet motio vel applicatio unius potentiae per aliam, quo modo potentia motiva existens in musculis excitatur vel applicatur per appetitum, et voluntas dicitur hoc modo movere omnes animae potentias ».
} 
d'une sympathie (sympathia) entre ces pouvoirs ${ }^{43}$. Cette sympathie exprime un ordre « horizontal» qui trouve son fondement «vertical» dans l'unité de la forme substantielle unique, sous-jacente à ces facultés. Selon Suárez, la coordination systématique entre les opérations et les différentes facultés d'un même être est en réalité le principal argument en faveur de l'existence et de l'unité de cette forme substantielle ${ }^{44}$. Il l'explique ainsi dans le cas des actions vitales d'un être vivant :

Davantage on peut produire un excellent argument contraire tiré de la connexion des opérations. Car lorsque l'intellect pense, la volonté est excitée à aimer, etc.; et ceci est dû au fait que la même âme opère à travers ces deux pouvoirs. Or le simple fait de l'enracinement habituel ou de l'émanation lointaine à partir d'une même âme ne suffirait pas à établir cette causalité, ou plutôt motion et excitation, actuelle, si chaque opération procédait actuellement de sa propre puissance seulement sans une connexion dans quelque principe commun. De là vient également cette dépendance entre l'appétit vital et la cognition, en sorte que rien ne puisse être aimé qui ne soit d'abord connu ${ }^{45}$.

Ainsi, en dépit du fait que chaque faculté manifeste une activité spécifique, ces activités ne fonctionnent pas de manière désordonnée ou aléatoire. Bien au contraire, la volonté veut un objet seulement lorsque l'intellect a formé une représentation de cet objet comme un bien. Cette coordination exige un fondement qui consiste dans le fait que toutes ces activités enveloppent l'action causale d'un unique agent, la forme substantielle. Le rôle causal exact de cette forme est précisée dans la partie centrale de ce passage. Suárez rejette une première explication, selon laquelle l'influence de l'âme se cantonne au fait qu'elle soit la source d'où découle l'existence des facultés, ce qu'il nomme un « enracinement habituel» (habitualis radicatio). Celui-ci permet bien de rendre compte du caractère ordonné des facultés ellesmêmes. Ainsi il ne peut exister une âme seulement pourvue d'une faculté conative sans une faculté cognitive d'un niveau correspondant : l'appétit sensible présuppose l'existence d'une

\footnotetext{
${ }^{43}$ Les expressions apparentées de consensio, co-ordinatio, consonantia ou encore sympathia reviennent régulièrement sous la plume de Suárez; $c f$. CDA II.5.5 ; III.7.11 ; VI.2.10 ; VI.2.13 : " est ergo haec naturalis consensio inter has potentias, quod eo ipso quod anima aliquid visu percipit, statim sonat similitudinem illius rei in imaginatione sua »; IX.2.12 ; XXII.2.29; XXIII.1.11; 5.14; 7.2. La notion a attiré l'attention des commentateurs ; voir (Ludwig 1929), (Spruit 1995, vol.2, 294-307) et (Knuuttila 2014, 196sq.).

${ }^{44}$ DM XV.1.7 : « ex eadem hominis compositione colligitur aggregationem plurium facultatum vel formarum accidentalium in simplici subiecto substantiali non satis esse ad constitutionem rei naturalis; nam in homine sunt illae facultates et formae accidentales plures fortasse ac perfectiores quam in aliis naturalibus rebus, et tamen non sufficiunt ad constitutionem alicuius naturalis entis completi, sed praeterea requiritur forma quae veluti praesit omnibus illis facultatibus et accidentibus et sit fons omnium actionum et naturalium motuum talis entis, et in qua tota illa varietas accidentium et potentiarum radicem et quamdam unitatem habeat $\gg$. Cet argument établit l'insuffisance d'un simple agrégat de facultés et justifie l'unité de la forme substantielle. Voir (Rozemond 2012) et (Shields 2012).

${ }^{45}$ DM XVIII.5.3 : «Praeterea sumitur a contrario optimum argumentum ex connexione operationum; nam dum intellectus intelligit, voluntas excitatur ad amandum, etc.; hoc autem ideo est quia eadem anima per utramque potentiam actu operatur, nam sola veluti habitualis radicatio seu remota dimanatio ab eadem anima non esset satis ad hanc actualem causalitatem seu motionem et excitationem, si unaquaeque operatio a sua sola potentia actualiter prodiret sine connexione in aliquo communi principio. Et inde etiam oritur dependentia inter appetitum vitae et cognitionem, ut nihil possit esse amatum nisi sit praecognitum ».
} 
faculté sensorielle, de même que l'appétit rationnel ou volonté présuppose l'existence d'un intellect ${ }^{46}$. Cependant cet ordre entre les facultés ne suffit pas à rendre compte du caractère ordonné des activités de ces facultés ${ }^{47}$. La dépendance fonctionnelle entre les activités des différentes facultés provient en effet de ce qu'une même forme substantielle est la cause efficiente des actions de ces facultés.

Suárez précise ce qu'il entend par la dépendance de la volonté par rapport à un bien représenté par l'intellect :

Nous pouvons plus facilement éviter ces problèmes en niant que la connaissance concoure activement avec l'acte de l'appétit et en maintenant qu'elle est seulement une condition représentant l'objet telle qu'une fois posée, l'appétit forme son propre acte par la sympathie naturelle de ces facultés. Et à cela contribue le fait que ces facultés soient enracinés dans la même âme. L'âme, ou le sujet par elle, est ce qui opère principalement et ce qui utilise ces facultés. Et ainsi, pendant qu'elle perçoit par l'une un objet qui lui convient, elle le désire par une autre. Non certes qu'à travers l'une elle soit une cause efficiente dans l'autre, mais parce qu'à partir d'un objet appréhendé par l'une, elle est excitée à opérer par l'autre. Et cette excitation ne se fait pas par un changement réel et effectif, mais par un changement métaphorique ou final, et ainsi elle ne requiert pas de proximité locale, mais « animale » pour ainsi $\operatorname{dire}^{48}$.

Il faut ainsi distinguer la relation de l'âme aux opérations de ses facultés de la relation entre les opérations de ces mêmes facultés. La première relève du régime de la cause efficiente: l'âme, ou bien le suppositum humain par l'intermédiaire de l'âme, est cause efficiente de l'acte d'intellection aussi bien que de la volition. Elle en est le principe principal ut quod. La seconde relation met en jeu la sympathie entre ces facultés. Ce passage met en lumière l'impossibilité d'inférer du fait que l'âme est la cause efficiente d'un acte d'intellection et qu'elle est la cause efficiente d'un acte de volition à un moment ultérieur que le premier est une cause efficiente du second. Le rapport entre les deux relève d'un autre régime de causalité, celui de la cause finale qui implique un «changement métaphorique » ${ }^{49}$. Ailleurs, Suárez parle d'un «mouvement objectif» pour désigner l'influence d'une

\footnotetext{
${ }^{46} \mathrm{DM}$ XV.1.14: « in uno ente naturali multae proprietates coniunguntur, quae interdum ita sunt inter se subordinatae ut una ab altera oriatur, ut voluntas ab intellectu ».

${ }^{47}$ Toutefois, dans certains passages Suárez admet qu'une telle habitualis radicatio suffit à rendre compte de l'ordre des activités, comme dans CDA III.2.8 : «Et ideo ad illam immediate ordinatur, sicut eadem anima immediate est receptiva intellectus et voluntatis, et quodammodo factiva, nam a substantia illius profluunt immediate illae potentiae $»$. Cela ne reflète cependant pas sa thèse définitive.

${ }^{48}$ DM XVIII.8.40 : «Et ideo nos facilius has angustias evademus negando notitiam concurrere active ad actum appetitus, sed tantum ut conditionem repraesentantem obiectum, qua posita, appetitus efficit suum actum per naturalem sympathiam harum potentiarum. Et ad hoc optime confert radicatio illarum facultatum in eadem anima; illa enim, seu suppositum per illam, est quod principaliter operatur et utitur his facultatibus, et ideo, dum per unam percipit obiectum sibi conveniens, per aliam illud appetit; non quidem quia per unam efficiat in aliam, sed quia ex obiecto per unam apprehenso excitatur ad operandum per aliam, quae excitatio non est per immutationem realem et effectivam, sed per metaphoricam seu finalem, et ideo non requirit localem propinquitatem, sed animalem, ut sic dicam ».

${ }^{49}$ Suárez rattache l'idée de mouvement métaphorique à Aristote (De la génération et de la corruption I.7, 324b14-15) et à Thomas d'Aquin (ST I-II, q.1, a.1).
} 
représentation sur une volition et définit à par son intermédiaire la causalité d'une cause finale : «la causalité d'une fin consiste dans le mouvement métaphorique de la volonté par lequel la fin attire à elle la volonté $\rangle^{50}$. Le caractère métaphorique de la causalité finale ne signifie pas une déréalisation de la cause finale, mais qu'elle décrit une tendance provenant de la représentation d'un objet comme un bien ${ }^{51}$. Cependant, ce qui produit la volition n'est pas le bien représenté, mais l'agent lui-même qui est la cause efficiente à la fois de cette représentation et de cette volition. Un aspect important de la conception suarézienne est que la causalité finale, c'est-à-dire le mouvement métaphorique du bien représenté par l'intellect, n'existe pas avant l'exercice de la causalité efficiente de la volonté dans la production d'une volition :

[On dira] qu'une seule et même action de la volonté est causée par la fin et par la volonté elle-même. Et en tant qu'elle provient de la volonté, elle est une causalité efficiente; et en tant qu'elle provient de la fin, elle est une causalité finale. Et en vertu de la première elle est un mouvement réel et propre, parce qu'une telle action émane de cette puissance comme d'un principe physique propre, mais en vertu de la seconde, elle est un mouvement métaphorique, parce qu'elle émane d'un objet qui charme et attire à soi la volonté . $^{52}$

Suárez semble défendre une théorie du double aspect de la causalité des actions intentionnelles : une seule et même action de la volonté peut en effet être considérée sous deux aspects : (i) en tant qu'elle est l'action d'une cause efficiente, autrement dit de la forme substantielle opérant par l'intermédiaire de la volonté ; (ii) en tant qu'elle est l'action d'une cause finale, c'est-à-dire du bien représenté par l'intellect ${ }^{53}$. Mais si l'agent ne cause pas la volition, cette représentation de l'intellect ne meut en aucun sens l'intellect. La force motivationnelle n'est pas une caractéristique inhérente à la représentation d'un bien, mais quelque chose qu'elle acquiert en vertu de l'action de l'agent lui-même.

\footnotetext{
${ }^{50}$ DM XXIII.5.2 : « ... Causalitas finis consistit in motione metaphorica voluntatis, qua illam ad se allicit...». Un des éléments décisifs de la théorie de la cause finale est qu'elle ne peut agir sans une représentation de la fin. Cela semble réduire la téléologie aux êtres pourvus de capacités cognitives. Les agents naturels dépourvus de cognition comme un rocher, ne sont pas directement soumis à la causalité finale, mais seulement en tant qu'ils sont intégrés à un ordre providentiel voulu par Dieu.

${ }^{51}$ DM XXIII.1.14. Il y a un débat entre les commentateurs sur le statut subordonné ou non de la finalité par rapport à l'efficience. La thèse classique est que Suárez rompt avec la tradition thomiste en faisant de la cause efficiente le modèle de la causalité et en subordonné la finalité à l'efficience. Cette thèse est défendue notamment par (Carraud 2002) ou (Schmaltz 2008, 29-34). Elle est critiquée par (Penner 2015) qui soutient (i) le caractère réel de la causalité finale, et (ii) l'absence de subordination à la causalité efficiente. Dans l'analyse de l'action humaine du moins, il semble cependant qu'il y ait une intégration de deux aspects de la causalité.

${ }^{52}$ DM XXIII.4.8 : « ita aiunt unam et eamdem actionem voluntatis causari a fine et a voluntate ipsa, et prout est a voluntate esse causalitatem effectivam, prout vero est a fine esse causalitatem finalem, et priori ratione esse motionem realem ac propriam, quia talis actio manat a potentia ut a proprio principio physico, posteriori autem ratione esse motionem metaphoricam, quia manat ab obiecto alliciente et trahente ad se voluntatem ».

${ }^{53}$ DM XXIII.4.9 : «actio voluntatis simul ac fit, necessario pendet a voluntate et a fine; illa ergo eadem actio quatenus est a voluntate est dependentia activa ab illa, quatenus vero est a fine, est dependentia finalis... bonum autem voluntati propositum solum finaliter concurrit ad actum eius, quia solum movet metaphorice attrahendo propositum per cognitionem, etiamsi aliter in re non existat ». Cf. DM XXIII.4.12-13.
} 


\section{La réponse aux problèmes de la rationalité et de la chance}

Ce long détour par l'analyse des pouvoirs causaux, du rôle de la forme substantielle dans la causalité efficiente, enfin de la sympathie à l'œuvre dans les actions vitales est indispensable si l'on veut ressaisir la réponse de Suárez aux principales objections, l'objection de la rationalité et celle de la chance, qui menacent entre autres les théories de la causalité de l'agent. En un mot, selon la première, le choix n'est pas contrôlé par l'agent parce qu'il est sans raison. Selon la seconde, le choix n'est pas contrôlé par l'agent parce qu'il n'est pas causé, ou du moins parce que sa production par l'agent est elle-même dépourvue de cause ${ }^{54}$.

Ces problèmes, centraux dans les débats contemporains sur le libre-arbitre, sont confrontés par Suárez à l'occasion d'une discussion sur l'influence du jugement pratique issu de l'intellect sur la volonté ${ }^{55}$. D'après lui, le choix d'une action visant un objet $X$ pris pour fin suppose un jugement antécédent selon lequel $X$ est un bien. Néanmoins la présence de ce jugement ne nécessite pas le choix :

Bien que l'objet comme tel ne détermine pas la volonté, il peut cependant être suffisant pour l'exciter et l'attirer, afin que, par sa propre liberté, elle se détermine et se porte vers lui, parce que si quelque bien (ratio boni) est représenté dans l'objet, il est de soi suffisant à mouvoir la volonté; donc cette détermination n'est pas nécessaire pour que la volonté se meuve (moveatur) ${ }^{56}$.

Un slogan résume cette thèse : « la volonté est déterminée par l'intellect par rapport à la suffisance, mais elle se détermine elle-même par rapport à l'efficace $»^{57}$. La terminologie de la suffisance et de l'efficace est empruntée aux discussions sur la grâce. Le jugement de l'intellect est suffisant en ce sens qu'il est un réquisit indispensable au choix de la volonté : il est nécessaire que quelque objet soit présenté comme un bien pour que l'agent ait une raison de le vouloir. Le texte précédent ajoute qu'il suffit à mouvoir la volonté, autrement dit que le

\footnotetext{
${ }^{54}$ Pour ces deux types d'objections, voir (Michon 2011, 116-117) et (Pereboom 2014, chap.3).

${ }^{55}$ Voir la discussion approfondie dans (Penner 2013, 18-26).

${ }^{56}$ DM XIX.6.9: «Quamvis autem obiectum non sic determinet voluntatem, potest esse ita sufficiens ad excitandam et alliciendam illam ut ipsa sua libertate determinetur aut feratur in illud, quia si in obiecto repraesentatur aliqua ratio boni, illa est de se sufficiens ad movendam voluntatem; ergo illa determinatio necessaria non est ut voluntas moveatur ». Il y a cependant deux exceptions. La première concerne la liberté de spécification par rapport au bonum in commune. Une seconde exception concerne la vision de Dieu aux cieux qui supprime la liberté d'exercice (cf. DM XIX.8.15). Face au bonum commune, la volonté préserve le pouvoir de vouloir le contraire et l'agent est libre. Dans le cas de la vision béatifique en revanche, l'adhésion est volontaire mais non libre en raison de l'absence d'un pouvoir des contraires.

${ }^{57}$ DVI VIII.4.11, OO IV, 264 : « Ab intellectu determinatur quasi quoad sufficientiam ; ipsa vero se determinat quoad efficaciam ». Comme le remarque S. Penner, la terminologie de la sufficientia porte la marque d'un héritage scotiste (Penner 2013, 26). Selon Duns Scot en effet, la volonté est caractérisée par une indétermination qui est une perfection porteuse d'une «surabondance de suffisance». Cf. Jean Duns Scot, Quaestiones super libros Metaphysicorum Aristotelis, IX, q.15, Opera philosophica, IV, 683 : « est alia [quaedam indeterminatio] superabundantis sufficientiae, quae est ex illimitatione actualitatis, vel simpliciter vel quodammodo ».
} 
jugement pratique constitue un motif prima facie pour la volonté. Mais ce jugement n'est pas efficace en ce qu'il ne nécessite pas ce choix, qui dépend ultimement de la volonté.

Suárez établit ce point en envisageant trois cas de figures distincts. Le premier (C1) couvre les cas dans lesquels l'agent considère un unique jugement pratique selon lequel $X$ est un bien. Le second concerne ceux dans lesquels il y a un jugement comparatif sur la valeur de plusieurs objets. Ce second cas de figure se subdivise en outre en deux cas de figure distincts. Dans un premier cas (C2), ce jugement comparatif présente des objets d'une valeur approximativement égale. Une situation comparable à celle de l'âne de Buridan en est un exemple typique. Mais Suárez lui préfère l'indifférence de la volonté divine face aux différentes créatures qu'il aurait pu créer. Enfin, dans le second cas de figure (C3), un jugement comparatif représente l'une des options (soit entre plusieurs fins à poursuivre, soit entre plusieurs moyens en vue de cette fin) comme incontestablement meilleure.

Dans un passage de la section 6 de la dispute XIX, Suárez défend sa position contre une objection thomiste selon laquelle dans les cas (C1), la volonté est nécessitée par le jugement pratique, faute d'alternative vers laquelle elle peut se tourner. Sa réponse consiste à ériger la situation d'indifférence de type $(\mathrm{C} 2)$ en paradigme de la liberté de la volonté et à défendre ainsi l'indifférence de la volonté dans les deux autres cas de figure :

\begin{abstract}
Mais on objectera : si un seul jugement est présent, la volonté le suivra complètement, parce qu'elle n'a pas autre chose vers quoi se tourner. Et s'il y a plusieurs jugements, elle suivra nécessairement celui qui porte sur le moyen le plus utile ou l'objet le meilleur, et ainsi, elle sera toujours déterminée par le jugement. On répond à cela en premier lieu qu'il peut y avoir des jugements sur des moyens égaux, et alors la volonté ne pourra être déterminée par le jugement et elle ne restera pas nécessairement en suspens, mais telle est sa liberté qu'elle pourra choisir l'une et omettre l'autre. Et si elle ne le peut pas, je ne vois pas comment elle peut être dite libre. Mais en réalité, elle le peut, parce qu'elle est libre en telle sorte qu'elle peut vouloir ce qui est bon et ne pas vouloir ce qui n'est pas nécessaire. Et la liberté divine en est un modèle splendide, elle qui, entre cette matière et une autre parfaitement égale et semblable, et entre ces cieux et d'autres parfaitement égaux (et ainsi des autres choses), a choisi les uns plutôt que les autres. Or on ne peut le rapporter à un jugement inégal, parce qu'il n'y a nulle raison, ni de leur côté, ni du côté de Dieu, qui pourrait fonder la vérité d'un tel jugement. Donc ce choix préférentiel provient seulement de la libre détermination de la volonté divine. Derechef, même lorsque les objets ou les moyens sont jugés inégaux, je pense qu'il est plus probable (quoique moins certain), que la volonté n'est pas déterminée nécessairement à ce qui est le meilleur par la force du jugement. Cela est prouvé de ce que, par cela même qu'aucun n'est proposé [à la volonté] comme nécessaire, la volonté peut ne pas aimer l'un et l'autre. Donc elle peut aussi indifféremment aimer n'importe lequel d'eux, et rejeter l'autre. Et d'après de nombreux auteurs, cela semble nécessaire dans la volonté divine, parce qu'il a pu faire de meilleures choses que ce qu'il a fait, que pourtant il n'a pas voulues ${ }^{58}$.
\end{abstract}

\footnotetext{
${ }^{58}$ DM XIX.6.13 : «Sed urgebis: nam si unum tantum adest iudicium, illud omnino sequetur voluntas, quia non habet aliud ad quod se vertat. Si vero adsunt plura, necessario sequetur illud quod est de utiliori medio aut meliori obiecto, atque ita semper determinabitur a iudicio. Respondetur, imprimis, posse esse iudicia de mediis aequalibus, et tunc voluntas nec poterit determinari a iudicio nec necessario erit suspensa, sed haec est eius libertas ut unum possit eligere et aliud omittere. Quod si hoc non potest, non video quomodo possit dici libera;
} 
Dans le cas (C2) La volonté est alors absolument libre de choisir l'une ou l'autre des options présentées comme approximativement égales par l'intellect. Dans les situations (C3), la volonté peut choisir à l'encontre de ce que l'intellect lui présente comme le meilleur, même si la plupart du temps elle choisira conformément à ce jugement. Enfin, Suárez étend cette analyse au cas (C1). La volonté n'est pas nécessitée à choisir $X$ parce qu'elle peut suspendre son action et mouvoir l'intellect à considérer un objet différent ou à réévaluer la valeur de $X$ :

Même si l'intellect pense seulement à un objet et juge qu'il est convenable et digne d'être désiré, et même s'il peut d'autant inviter pratiquement la volonté à le désirer, la volonté peut encore ne pas l'aimer en vertu de sa liberté, parce que pour précisément ne pas exercer un acte elle n'a pas besoin d'un autre jugement, mais qu'il suffit que, en vertu de l'état présent de l'homme, il ne juge pas que ce bien doive ici et maintenant être aimé de lui nécessairement. De là vient que la volonté peut alors ou bien suspendre l'acte, ou bien détourner l'intellect de penser à cet objet, ou bien l'appliquer afin qu'il s'enquière avec plus de soin pour savoir quelle est la bonté d'un tel objet et si une quelque malice ou inconvenance lui est attachée par laquelle elle puisse non seulement ne pas l'aimer, mais également le haïr. Jamais donc le jugement comme tel ne détermine absolument la volonté à moins que par ailleurs l'objet n'ait cette force en raison de l'excellence de sa bonté ${ }^{59}$.

La volonté peut donc exercer sa liberté d'exercice en suspendant son acte, mais également sa liberté de spécification dès lors que l'intellect réévalue son jugement. Un unique jugement pratique actuel suffit à garantir la liberté d'exercice, alors qu'une pluralité de jugements pratiques possibles est requise pour la liberté de spécification. On comprend dès lors pour quelle raison face au bien en général (bonum in commune), la volonté ne possède qu'une liberté d'exercice, étant donné qu'aucun jugement pratique hypothétique ne pourrait conduire à réévaluer à la baisse la valeur de ce bien.

Suárez ajoute qu'une pluralité de jugements possibles proposant des alternatives $B, C, \ldots$ à un bien donné $A$ suffit à garantir la liberté de spécification de la volonté même si $A$ possède une valeur supérieure à $B, C, \ldots$ Mais cette possibilité ouvre une brèche dans laquelle peut

sed revera id potest, quia ad hoc est libera ut possit velle quod bonum est et non velle quod necessarium non est. Estque illustre exemplar huius libertatis voluntas divina, quae inter hanc materiam et aliam omnino aequalem et similem, et inter hos caelos et alios omnino aequales (et sic de aliis rebus), hos elegit creare prae aliis, quod non potest referri in iudicium inaequale, quia neque ex parte ipsorum nec ex parte Dei est aliqua ratio qua possit fundari tale iudicium quod verum sit; est ergo illa praeelectio solum ex determinatione libera divinae voluntatis. Rursus etiam quando obiecta vel media iudicantur inaequalia, censeo probabilius (licet minus certum) non determinari voluntatem necessario ad id quod est melius ex vi iudicii. Probatur, quia, hoc ipso quod neutrum proponitur ut necessarium, potest voluntas utrumque non amare; ergo etiam potest indifferenter amare quodlibet illorum, praetermisso alio. Et in divina voluntate multis videtur hoc necessarium, quia potuit meliora facere quam fecit, et tamen noluit».

${ }^{59}$ DM XIX.6.14 : «Etsi intellectus de uno tantum obiecto cogitet, et illud iudicet esse conveniens et dignum ut appetatur, et quantum potest practice invitet voluntatem ut illud appetat, adhuc potest voluntas pro sua libertate illud non amare, quia ad non exercendum actum praecise non indiget alio iudicio, sed satis est ut per illud quod homo nunc habet non iudicet hoc bonum hic et nunc esse sibi necessario diligendum. Unde potest tunc voluntas vel suspendere actum vel divertere intellectum ne de illo obiecto cogitet, vel applicare illum ut de tali obiecto diligentius inquirat quanta sit eius bonitas, et an habeat coniuctam aliquam malitiam vel disconvenientiam, ob quam possit non solum non amare illud, sed etiam odisse. Nunquam ergo iudicium ut sic determinat omnino voluntatem nisi alioqui obiectum ob excellentiam bonitatis eam vim habeat $»$. 
s'engouffrer l'objection de la rationalité. Celle-ci repose sur le postulat que l'explication par les raisons doit être rattachée à la motivation: le jugement selon lequel $X$ est un bien s'accompagne d'une motivation à vouloir $X$ et donne en même temps une raison de vouloir $X$. Si l'on admet que les raisons de l'action sont proportionnées à la valeur des biens représentés, il s'ensuit que la liberté de la volonté dans les cas (C2) suppose la possibilité d'agir à l'encontre des raisons. Réciproquement, lorsque les biens représentés sont de valeur égale (C3), il semble que la volonté agisse sans raison. Enfin, dans les cas (C1), il semble que la liberté de la volonté soit incompatible avec l'exigence que les raisons de l'action soient contrastives. En somme, l'objection de la rationalité contre une théorie incompatibiliste à la manière de Suárez repose sur deux prémisses. L'une est que toute explication par les raisons est de nature contrastive, autrement dit, qu'une explication rationnelle d'un état de choses $p$ doit permettre en même temps d'expliquer pourquoi $p$ plutôt que n'importe quel autre état de choses $q$. La seconde prémisse est que les raisons ont une force motivationnelle intrinsèque. En d'autres termes, si j'ai une raison de vouloir quelque chose, je suis motivé à vouloir cela et la force de ma motivation donne la mesure de l'importance de la raison. Cette seconde prémisse implique en particulier que la volonté ne contrôle pas ce qui fait qu'une raison donnée constitue un motif.

Suárez rejette les deux prémisses de l'objection. À l'encontre de la première, il oppose une conception affaiblie de la raison pratique. L'idée est qu'une explication en termes de raison exige seulement que l'agent trouve quelque chose de bien dans l'action envisagée :

Car de ce que la volonté ne peut être mue vers un objet inconnu, il suit seulement qu'il est nécessaire qu'il y ait un jugement de l'intellect tel que la volonté peut choisir, mais il ne suit pas que ce jugement détermine la volonté à un choix unique ${ }^{60}$.

Suárez s'oppose ici à une conception stricte de la rationalité pratique selon laquelle le choix de $A$ requiert un jugement comparatif tout bien considéré indiquant que $A$ est meilleure que toute option envisageable dans cette circonstance. Cela entraînerait en effet la nécessitation de la volonté par le jugement pratique qu'il rejette précisément. Ce qui est requis c'est un jugement « tout choses considérées » (par contraste avec un jugement prima facie) selon lequel $A$ est un bien :

Donc ce qui suffit c'est un jugement par lequel tel moyen est jugé utile et, toutes choses considérées, apte à être choisi. Car, de même qu'afin que la vue puisse se porter sur un objet il n'est pas nécessaire qu'elle lui soit appliquée comme vu ou comme devant être vu, mais comme visible, de même, afin que

\footnotetext{
${ }^{60}$ DM XIX.6.10 : «EX eo enim quod voluntas non potest ferri in incognitum, solum habetur necessarium esse iudicium intellectus ut voluntas possit eligere, non vero sequitur oportere ut illud iudicium determinet voluntatem ad unum ».
} 
la volonté soit mue par un objet, il suffit qu'il lui soit proposé comme aimable, même s'il n'est pas jugé comme devant être absolument choisi ${ }^{61}$.

Ainsi lorsque l'agent est confronté à des raisons d'un poids inégal, il y a une explication rationnelle de l'action simplement parce qu'un jugement pratique présente l'action comme possiblement voulue. Par exemple, je peux préférer rester chez moi plutôt que me rendre à l'opéra alors que je dispose d'un billet pour la représentation du soir : bien qu'il semble plus rationnel dans ces circonstances de me rendre à l'opéra, le choix de rester à la maison n'est pas irrationnel pour autant, dès lors que je peux envisager cette décision d'une certaine façon comme un bien. On pourrait objecter que ce critère semble suffisamment faible pour que pratiquement toute action puisse être tenue pour raisonnable. Toutefois Suárez ne considère pas que dans des circonstances données n'importe quel choix est raisonnable et c'est pour cette raison qu'il ajoute la clause omnibus pensatis («toutes choses considérées »). Un individu donné, Marie peut ainsi juger prima facie agréable de boire un verre de vin au déjeuner, mais estimer après délibération, qu'étant donné son intention de travailler dans l'après-midi, elle devrait y renoncer. Ainsi, l'indifférence de la volonté s'exerce sur une classe restreinte d'actions possibles, restriction provenant notamment des intentions préalables de l'agent, comme dans l'exemple précédent, mais aussi de considérations d'ordre moral que Suárez enveloppe dans la notion de raison, si on modifiait l'exemple précédent en indiquant qu'il s'agit d'un jour de Carême et que Marie est croyante. Mais selon Suárez, cette classe n'est, dans les cas ordinaires, jamais restreinte à une unique action possible et il est dès lors toujours possible d'agir à l'encontre de la raison prévalente. Suárez rejette ainsi l'hypothèse selon laquelle toute explication par les raisons est de nature contrastive.

Il rejette également la seconde prémisse de l'objection de la rationalité. Si les jugements pratiques étaient intrinsèquement motivationnels, alors en choisissant à l'encontre d'un jugement représentant une action comme la meilleure action possible, l'agent ferait preuve d'irrationalité. On peut reformuler cette objection sous la forme d'un dilemme : si l'on dit que le poids des raisons est indépendant du pouvoir de l'agent, alors ou bien ce pouvoir découle de ces raisons et il est causalement inerte, ou bien il « fait la différence » dans certains cas, mais alors ce choix n'est pas rationnel. Au contraire, Suárez soutient que c'est seulement à travers le choix volontaire que ce qui est représenté comme un bien par l'intellect acquiert une force motivationnelle :

\footnotetext{
${ }^{61} \mathrm{ibid}$. : « Sufficit ergo illud iudicium quo medium hoc iudicatur utile et, pensatis omnibus, aptum ut eligi possit; et idem est de quolibet obiecto bono, quod, scilicet, iudicetur sufficienter bonum ut amari possit. Nam sicut, ut visus ferri possit in obiectum, non est necesse quod applicetur ut visum vel ut videndum, sed ut visibile, ita, ut voluntas moveatur ab obiecto, satis est quod proponatur ut diligibile, quamvis non iudicetur omnino eligendum ».
} 
Davantage, notre expérience montre que même une fois que nous connaissons la menace d'un châtiment ou la promesse d'une récompense, il est en notre pouvoir d'être mû ou de ne pas être mû par une telle raison ${ }^{62}$.

Bien que l'objet comme tel ne détermine pas la volonté, il peut être suffisant pour l'exciter et le stimuler de telle sorte que, par sa propre liberté elle se détermine par elle-même ou se porte d'elle-même vers cet objet, parce que si quelque aspect de bonté est représenté dans l'objet, il est suffisant à mouvoir la volonté. Ainsi cette détermination est nécessaire pour que la volonté soit mue ${ }^{63}$.

Dans ces deux passages, Suárez souligne que si les raisons influencent notre choix, ce n'est pas par l'intermédiaire d'une causalité efficiente, mais par le mouvement métaphorique caractéristique de la causalité finale. Cette influence métaphorique renferme ainsi deux aspects : (i) une composante représentationnelle et normative - la représentation d'un objet comme bon à certains égards; (ii) une composante motivationnelle, par laquelle cet objet motive la volonté à choisir cet objet. Cette composante motivationnelle n'est pas intrinsèque à la représentation, mais dépend de l'agent ${ }^{64}$. C'est ce que recouvre la notion de consensus ou consentement $^{65}$. En effet, le jugement pratique ne motive la volonté qu'à la condition que la volonté poursuive une certaine fin à travers cet objet, même si cette fin n'est pas toujours voulue formellement ou expressément, mais souvent virtuellement :

Je dis en premier lieu que par le choix, en tant que choix, la fin est toujours d'une certaine façon voulue, bien que parfois elle soit voulue virtuellement ${ }^{66}$.

Chaque acte de la volonté engage une détermination et une éventuelle réévaluation de cette fin. Il s'ensuit que la poursuite d'une fin (intentio finis) est contenue virtuellement dans tout choix d'un bien particulier :

Car on suppose assurément que celui qui choisit entre un bien honnête et un bien plaisant est affecté par le bien comme tel, autrement il ne choisirait pas aussitôt entre ces biens spécifiques, mais il devrait préalablement décider s'il doit poursuivre le bien. Et il en va ainsi dans les cas semblables. Ainsi donc, la tendance vers une fin particulière (en tant qu'elle est un mouvement vers un objet particulier en vertu

\footnotetext{
${ }^{62}$ DM XIX.2.15 : « Praeterea experimur etiam post cognitam comminationem poenae aut promissionem praemii situm esse in potestate nostra ea ratione moveri aut non moveri; idemque est de precibus, exhortationibus et similibus excitationibus ».

${ }^{63}$ DM XIX.6.9 : «Quamvis autem obiectum non sic determinet voluntatem, potest esse ita sufficiens ad excitandam et alliciendam illam ut ipsa sua libertate determinetur aut feratur in illud, quia si in obiecto repraesentatur aliqua ratio boni, illa est de se sufficiens ad movendam voluntatem; ergo illa determinatio necessaria non est ut voluntas moveatur ».

${ }^{64}$ Notre interprétation diffère sur ce point de celle que défend (Pink 2012). D’après ce dernier, Suárez développe un modèle des raisons pratiques en termes de forces (force model) : ces raisons pratiques ne causent pas l'action externe, pas plus que la décision volontaire. Mais elles imposent une exigence, analogue sur le plan pratique de la force d'un argument. Notre lecture s'éloigne de celle de Pink en ce que nous pensons que les raisons n'imposent pas d'elles-mêmes une telle exigence, mais seulement à condition d'être choisies par l'agent (la forme substantielle ou l'âme), lequel est la cause (agente) du choix.

${ }^{65}$ DVI VIII.2, p.257b : «Iudicium intellectus antecedit voluntatis actum, et est quasi naturalis. In potestate autem voluntatis est, illi assentiri, vel non; et ideo illa dum libere operatur est quae perficit consensum ». Sur la notion de consentement, voir Thomas d'Aquin, ST I-II, q.15, en particulier a.1.

${ }^{66}$ DVI VIII.3.3, OO IV, 259b: « Dico primo, per electionem, ut electio est, semper est aliquo modo volitus finis, quamvis interdum sit virtualiter amatus ».
} 
de sa propre bonté et vers d'autres objets en vertu de la bonté de cet objet lui-même), est un amour ou une intention de la fin. Malgré cela, en tant qu'elle est une action libre à travers laquelle ce bien est préféré à d'autres, elle est un libre choix de ce bien comme moyen ou du moins comme partie d'un moyen vers la félicité ultime, laquelle est visée par la volonté dans ses actions. Et l'action même de viser ou d'aimer cette félicité ultime, en tant qu'elle est exercée librement ici et maintenant, et qu'elle est préférée à l'absence de cette action ou à l'action de haïr la félicité, est un choix virtuel par lequel l'action qui est choisie ici et maintenant comme moyen en vue d'un commencement ou d'une obtention de la félicité. Et pour cette raison, on dit d'ordinaire que n'importe quelle intention d'une fin particulière est un choix virtuel de la félicité, en sorte qu'il n'est pas toujours nécessaire qu'elle soit précédée d'une intention expressément formée de la félicité, mais qu'il suffit d'une propension naturelle et nécessaire ${ }^{67}$.

L'acte de volonté qui porte sur un moyen inclut virtuellement un acte de volonté se rapportant à la fin. L'inclusion virtuelle signifie tout d'abord que cet acte se rapportant à la fin n'a pas besoin d'être explicitement formé dans toutes les circonstances. Il est le plus souvent implicite. Ainsi celui qui porte secours à quelqu'un qui se noie accomplit une action altruiste sans avoir besoin de former un premier acte d'adhésion au bien honnête : celle-ci est virtuellement comprise dans l'intention de porter secours au noyé, de même que cette dernière est incluse dans les différentes actions entreprises en vue de cette fin. Mais l'inclusion virtuelle fonctionne également à rebours : la liberté à l'égard du choix des moyens ou de la sélection d'une fin particulière implique la liberté à l'égard de la fin elle-même. C'est par le choix de son action particulière que l'agent détermine un ordre entre les différentes fins qu'il peut poursuivre. Ce faisant, ce même choix détermine la force motivationnelle des raisons de son action. En règle générale, le choix se conforme à des fins déterminées antérieurement au choix lui-même et susceptibles d'êtres explicitement formulées antérieurement à ce choix. Mais dans certains cas, notamment dans les dilemmes moraux, le choix final engage une réévaluation de ces fins et par là du poids des raisons guidant le choix. Reprenons l'exemple d'un secours porté à un noyé. On peut dire qu'avant le choix, il y a dans l'agent (entre autres) un jugement pratique $\mathrm{J} 1$ en faveur d'un choix égoïste conduisant au choix A1 de poursuivre son chemin et un autre jugement $\mathrm{J} 2$ en faveur du choix altruiste A2. Le choix par l'agent de A2 plutôt que A1 implique une plus grande importance accordée à la fin représentée dans J2 par rapport à celle que représente $\mathrm{J} 1$. Bien que les jugements soient formulés avant le choix

\footnotetext{
${ }^{67}$ DM XIX.8.20 : « Nam qui eligit inter bonum honestum et delectabile, certe supponitur affectus ad bonum ut sic, alias non statim eligeret inter specialia bona, sed prius secum statueret an bonum esset sibi prosequendum; et idem est in omnibus similibus. Sic igitur, licet tendentia in finem particularem, quatenus est motus in aliquod obiectum propter bonitatem suam et in alia propter ipsum, sit amor vel intentio finis, tamen, quatenus est actus liber quo hoc bonum praefertur aliis, est virtualis electio huius boni ut sit medium, vel certe pars felicitatis quam voluntas intendit in suis actibus. Et ipsemet actus intentionis vel dilectionis etiam ultimae felicitatis, quatenus hic et nunc exercetur libere et praefertur eius carentiae vel otio, est virtualis electio qua talis actus hic et nunc eligitur ut medium ad inchoandam vel obtinendam felicitatem. Atque hac ratione dici solet omnem intentionem finis particularis esse virtualem electionem felicitatis, ad quam non semper est necesse ut praecedat intentio elicita ipsius felicitatis, sed sufficit naturalis ac necessaria propensio $»$.
} 
lui-même, leur force motivationnelle est déterminée par celui-ci, ce qui est possible parce qu'ils sont virtuellement inclus dans le choix. Pour le dire autrement, la valeur ou le poids motivationnel d'un bien n'est définitivement fixé qu'à travers le choix et c'est ce dernier qui révèle la nature du jugement toutes choses bien considérées (omnibus pensatis).

Cette solution à l'objection de la rationalité repose in fine sur la métaphysique de l'âme et de ses facultés et de leur rôle causal respectif. En effet, pour rendre compte du fait que la volonté est d'une certaine façon antérieure et postérieure à la fois au jugement pratique, Suárez invoque la sympathie naturelle des facultés ${ }^{68}$. À première vue, ce recours à l'idée de sympathie entre le jugement pratique et le choix actuel revient à postuler une sorte d'harmonie préétablie ou une coïncidence brute entre les deux. Toutefois, comme nous l'avons vu, le cœur de la conception suarézienne de la sympathie est constituée par l'idée d'une harmonie fonctionnelle entre les facultés qui ne se réduit ni à une causalité efficiente directe ni à une coïncidence inexpliquée entre les raisons et le choix. Elle repose au contraire sur l'unité de la forme substantielle ou de l'agent sous-jacent à ces facultés. L'bojection de la rationalité présuppose ainsi une conception métaphysique selon laquelle l'âme et ses facultés constituent une entité composée pourvue d'une simple unité d'agrégation (unum per aggregationem). À l'encontre de cette conception, Suárez mobilise ici de manière décisive la thèse de l'unité métaphysique de l'âme ${ }^{69}$.

Suárez propose ainsi une solution élaborée au problème de la rationalité. L'argument de la chance ne fait pas l'objet d'une discussion aussi explicite. Il est néanmoins possible de reconstruire une réponse suarézienne à partir de différents éléments de sa théorie. Dans les discussions contemporaines, les adversaires de la théorie de la causalité de l'agent soulignent que cette théorie n'offre pas une solution plus satisfaisante à cette objection que les conceptions libertariennes alternatives, d'un indéterminisme conçu dans le cadre d'une conception événementielle de la causalité. En effet, dans une situation de choix, rien d'antérieur à la décision de l'agent ne détermine quel choix sera finalement fait. Par suite, rien ne permet de fonder la différence entre le choix de $A$ plutôt que de non- $A$. Ceci se traduit par

\footnotetext{
${ }^{68}$ DM XIX.7.12 : «Dices: si tale iudicium nullo modo antecedit consensum liberum, ergo non ponitur ut repraesentet voluntati obiectum, neque ut inducat illam ad consensum; ad quid ergo est necessarius talis actus? Respondetur videri necessarium primo ex naturali sympathia et consensione harum potentiarum, quae necessaria fuit ut homo possit melius exsequi quod faciendum decernit ».

${ }^{69}$ Une question subsidiaire consiste à accorder l'unité per se de l'âme avec la distinction in re de ses facultés. Pour certains commentateurs, il s'agit d'une distinction virtuelle: les facultés de l'âme n'existent pas actuellement en elle, mais seulement à travers leurs différentes manifestations. Telle est l'interprétation de (Shields 2014, 213-217). Pour d'autres commentateurs en revanche, Suárez défend une conception plus robuste de la réalité et distinction des facultés, n'échappant pas ainsi au risque d'une fragmentation de l'agent en un réseau complexe de modules plus ou moins indépendants les uns des autres. Telle est l'interprétation que suggère (Perler 2015).
} 
le fait que si la situation se répétait dans différents mondes possibles, l'agent choisirait $A$ dans certains d'entre eux et non- $A$ dans certains autres, sans différence entre les réquisits de l'action. Comment distinguer ce résultat d'une situation aléatoire? Une réponse habituelle du partisan de la causalité de l'agent consiste à dire que ce n'est pas purement dû au hasard et que l'agent exerce en réalité un authentique contrôle sur sa décision pour la raison que la causalité de l'agent est une forme sui generis de causalité impliquant un contrôle immédiat sur des actions singulières.

Le problème est qu'une réponse de cette nature ne peut être adoptée pour la théorie de Suárez. En effet ce dernier soutient que toute causalité efficiente relève d'une forme de causalité substantielle dont les causes libres ne forment qu'un cas parmi d'autres. Donc s'il y a des cas dans lesquels des causes non-libres présentent des processus indéterministes, il n'y a pas moyen de distinguer le contrôle exercé par l'agent libre sur ses actions de celui d'une substance naturelle sur les siennes. Or, dans la première section de la dispute XIX, Suárez s'attache précisément à rejeter la thèse selon laquelle il y a de la contingence au sens d'une indétermination réelle parmi les causes purement naturelles. Toutes les causes naturelles sont des causes nécessaires. Dans certains cas toutefois, il semble qu'on ait affaire à une indifférence véritable. Suárez donne des exemples tirés de la physique aristotélicienne : du feu qui se trouverait enfermé au centre de la Terre n'aurait pas plus de raison de s'élever dans une direction que dans une autre et pourtant il ne pourrait pas non plus se diffuser dans toutes les directions. Un second exemple est celui d'un morceau de verre parfaitement lisse posé sur une pierre parfaitement lisse et sur lequel tombe une autre pierre également lisse : il n'y aurait pas plus de raison que le verre se brise en un endroit plutôt qu'en un autre et pourtant il n'est pas possible que ce verre se brise entièrement. Un dernier exemple est celui de l'âne de Buridan, indifférent entre deux objets également désirables (DM XIX.1.5).

Les exemples d'un hypothétique indéterminisme naturel sont ainsi redécrits comme dus à notre ignorance de l'absence d'un des réquisits nécessaires à l'action de cette cause naturelle ${ }^{70}$. Lorsqu'on tient compte de l'ensemble complet des conditions ou réquisits, il en découle toujours un unique effet déterminé qui consiste parfois dans l'absence de l'effet attendu. Suárez répond ainsi à l'exemple du verre pris en étau entre deux pierres qu'il ne se brisera pas instantanément, parce que l'air situé entre la vitre et la roche ne peut être expulsé en un moment. Dans une telle situation, la cause naturelle à proprement parler n'agirait pas (DM XIX.1.7). Il résout ainsi le cas de l'âne de Buridan : d'une part, dans l'ordre naturel des

\footnotetext{
${ }^{70}$ DM XIX.1.6 : «non videtur carentia actionis oriri ex indifferentia, sed ex aliquo impedimento vel defectu alicuius conditionis necessariae ex supra positis $»$.
} 
choses, il y a toujours une différence entre deux biens désirables qui nécessite l'inclination de l'appétit sensible de l'animal. Mais d'autre part, même en admettant la possibilité d'un parfait équilibre, celui-ci serait incapable de se déterminer, précisément en raison de l'équilibre des motifs ${ }^{71}$. Il n'y a donc pas de causalité indéterministe en dehors des causes libres, c'est-à-dire des causes qui agissent à la lumière de raisons. Par suite, Suárez peut répondre à l'objection du hasard en maintenant qu'un agent libre exerce un contrôle spécifique sur son choix, et que ce dernier diffère d'un événement aléatoire, en vertu d'une double propriété que la cause libre est seule à posséder : un pouvoir des contraires guidé, mais non causé, par de raisons.

\section{Conclusion}

La conception suarézienne de la causalité libre présente ainsi une réponse à deux des principales objections à l'encontre des thèses libertariennes ainsi que des théories de la causalité de l'agent. Elle repose sur une notion, celle de sympathie naturelle, à première vue parfaitement ad hoc et obscure. Nous avons toutefois essayé de dissiper en partie cette impression en montrant que cette notion s'insère dans une conception complexe qui articule de manière systématique une théorie psychologique et une métaphysique de la causalité. Le concept de sympathie désigne le noyau essentiel de sa théorie des causes libres : à savoir que les raisons ne sont pas indépendantes des décisions volontaires, puisque leur force motivationnelle dépend de la volonté et plus fondamentalement de l'unique agent, la forme substantielle, d'où procèdent l'ensemble des opérations cognitives et appétitives ${ }^{72}$.

\footnotetext{
${ }^{71}$ DM XIX.1.9 : «Denique, si admittamus aequalem apprehensionem in omnibus circumstantiis, tam respectu appetitionis quam exsecutionis, concedendum videtur non esse in appetitu bruti facultatem ut se determinet ad unum potius quam ad aliud, ob carentiam libertatis. Unde illa indeterminatio non provenit ex indifferentia, sed potius ex naturali necessitate ac determinatione in modo operandi, et defectu conditionis necessariae ad sic operandum ».

${ }^{72}$ Il est éclairant de confronter la conception de Suárez à deux approches différentes de la causalité de l'agent développées récemment, par Randolph Clarke et Timothy O'Connor respectivement. Clarke propose ce qu'il appelle une explication intégrée (integrated account) selon laquelle une décision libre est causée par deux facteurs distincts : les raisons (un complexe croyances-désirs) et le pouvoir causal de l'agent (Clarke 2003, p.162). Ces deux facteurs contribuent conjointement à l'occurrence de l'action. Les raisons influencent l'agent en ce sens que lorsque l'agent $S$ cause (comme agent) la décision $A$, il agit en fonction d'une des raisons $R$. Le problème est d'expliquer cette influence non-causale des raisons sur l'agent. Clarke tente d'y répondre en postulant une correspondance entre les propensions de la cause-agente et du poids ou de la propension des raisons. Or une telle correspondance se heurte à deux objections. D'une part, elle introduit des coïncidences inexpliquées dans le monde. D'autre part, une telle correspondance n'est pas la relation appropriée pour rendre compte du rapport entre un agent et les raisons de son action. Le modèle récemment défendu par O’Connor diffère de celui de Clarke en ce qu'il soutient que les pouvoirs d'une cause agente sont non-causés, tout en étant structurés par les états motivationnels de l'agent. Autrement dit, le pouvoir de l'agent d'agir pour des raisons est inscrit dans le pouvoir causal lui-même, comme une cause structurelle. Les raisons fournissent à l'agent de nouveaux pouvoirs de choix et d'action, différentes raisons ayant le potentiel de motiver des actions différentes. Ces raisons sont ainsi des pouvoirs transitoires qui affectent temporairement le pouvoir causal de l'agent. Ainsi d'après le modèle de O'Connor, les raisons et le pouvoir causal de l'agent ne sont pas des entités distinctes. Les
} 
Nous avons enfin pu constater la présence d'une tension au sein de la philosophie de Suárez entre l'exigence d'unité de l'agent qui repose sur l'existence de formes substantielles immatérielles indivisibles qui sont la source immédiate de leurs différentes opérations d'une part et le réalisme des facultés mentales et des pouvoirs causaux d'autre part, qui semble aller dans le sens d'une fragmentation de l'âme. Cette fragmentation prête le flanc aux objections visant les théories libertariennes et en particulier à l'objection de l'agent évanescent (disappearing agent). Supposer des relations causales indéterministes entre des événements ou bien admettre dans le cadre d'une théorie substantialiste de la causalité des réseaux de pouvoirs causaux plus ou moins indépendants, cela revient à peu près au même. La leçon de notre étude est ainsi que la conception suarézienne des causes libres n'échappe pas totalement à cette tension entre deux tendances difficilement conciliables.

Abréviations :

CDA : Commentaria in de Anima (Suárez 1978-1991)

DM : Disputationes metaphysicae (Suárez 1856-1878, vols. 25-26)

DVI : De voluntario et involuntario (Suárez 1856-1878, vol.4)

$\mathrm{ST}$ : Summa theologiae

OO : Opera omnia

\section{Références :}

Anfray, J.-P., 2014, « Molina and Duns Scotus », in M. Kaufmann et A. Aichele (éds.), A Companion to Luis de Molina, Leyde, Brill, 325-364.

Carraud, V., 2002, Causa sive Ratio. La raison de la cause, de Suarez à Leibniz, Paris, Presses Universitaires de France.

Chisholm, R. M. 1976a, «The Agent as Cause », in Myles Brand et Douglas Walton (éds.), Action Theory, Dordrecht, Reidel, trad. franç. «L'agent en tant que cause », in M. Neuberg (dir.), Théorie de l'action, Bruxelles, Mardaga, p.225-240.

Chisholm R. M. 1976b, Person and Object, La Salle, Ill., Open Court.

Clarke, R., 2003, « Toward a Credible Agent-Causal Account of Free Will » in G. Watson (éd.), Free Will, Oxford, Clarendon, 285-298.

Duns Scot, Jean, 1950sq. Opera omnia (« édition vaticane »). éds. C. Balic et al., Vatican.

raisons sont au contraire un aspect du pouvoir causal de l'agent (O'Connor et Jacobs 2013 ; voir aussi la discussion dans Pereboom 2014). 
Duns Scot, Jean, 1997-2006, Opera philosophica, éd. G. J. Etzkorn, 5 vols., St. Bonaventure, NY, Franciscan Institute.

Kent, B., 1995, Virtues of the Will : the Transformation of Ethics in the Late Thirteenth Century, Washington D.C.

Knuuttila, S., 2014, "Suárez's Psychology» in V. Salas et R.L Fastiggi (éds.), A Companion to Francisco Suárez, Leyde, Brill, 192-220.

Locke, J., An Essay concerning Human Understanding, éd. P.H. Nidditch, Oxford, Clarendon, 1975.

Ludwig, J., 1929, Das akausale Zusammenwirken (sympathia) der Seelenvermögen in der Erkenntnislehre des Suarez, Munich, Ludwig.

Menn, S., 1997, «Suárez, Nominalism, and Modes », in K. White (ed.) Hispanic Philosophy in the Age of Discovery, Washington, DC, Catholic University of America Press, $226-56$

Michon, C. 2011, Qu'est-ce que le libre-arbitre ? Paris, Vrin.

Molina, L. de, 1595, Liberi arbitrii cum gratiæ donis, divina præscientia, providentia, prædestinatione et reprobatione concordia, Anvers ( $2^{\mathrm{e}}$ édition).

O'Connor, T. 2000, Persons and Causes. The Metaphysics of Free Will, Oxford, Oxford University Press.

O’Connor T. et Jacobs J.D., 2013, « Agent Causation in a Neo-Aristotelian Metaphysics » in S. Gibb, E.J. Lowe, R.D. Ingthorsson, Mental Causation and Ontology, Oxford, Clarendon, 173-192.

Ockham, Guillaume d', 1967-1989, Opera philosophica et theologica, St. Bonaventure, NY, Franciscan Institute.

Olivi, Pierre Jean, 1922-1926, Quaestiones in secundum librum Sententiarum, éd. B. Jansen, Quaracchi, 3 vols.

Pasnau, R., 2011, Metaphysical Themes. 1274-1671, Oxford, Clarendon.

Penner, S., 2013, «Free and Rational : Suárez on the Will », Archiv für die Geschichte der Philosophie 95 :1, 1-35.

Penner, S., 2015, «Final Causality: Suárez on the Priority of Final Causation », in J.L. Fink, Suárez on Aristotelian Causality, Leyde, Brill, 121-148.

Pereboom, D., 2014, Free Will, Agency, and Meaning in Life, Oxford, Clarendon.

Perler, D., 2015, "Faculties in Medieval Philosophy », in D. Perler (éd.), The Faculties. A History, Oxford, Oxford University Press, 97-139.

Pink, T., 2004, "Suarez, Hobbes, and the Scholastic Tradition in Action Theory », in The Will and Human Action: From Antiquity to the Present Day, T. Pink/M. W. F. Stone (éds.), Londres, Routledge, 127-153.

Pink, T., 2012, "Action and Freedom in Suárez's ethics» in Daniel Schwartz (éd.), Interpreting Suárez : Critical Readings, Cambridge University Press. 
Putallaz, F.-X., 1995, Insolente liberté. Controverses et condamnations au XIIIe siècle, Paris, Fribourg.

Rozemond, M., 2012, «Unity in the Multiplicity of Suárez' Soul », in B. Hill et H. Lagerlund (éds.), The Philosophy of Suárez, Oxford, Oxford University Press, 154-172.

Suárez, F., 1597, Disputationes metaphysicae, Salamanque, 2 vols. (repris dans OO vols. 25-26, texte électronique par S. Castellote Cubells et M. Renemann disponible sur http://homepage.ruhr-uni-bochum.de/Michael.Renemann/suarez/index.html).

Suárez, F., 1621, Partis secundae summae theologiae tomus alter, complectens tractatum secundum de opera sex dierum, ac tertium de anima, cui adiecti sunt elenchi admodum necessarii et utiles, éd. B. Alvarez, Lyon, repris dans OO, vol. 3.

Suárez, F., 1628, Ad Primam secundæ D. Thomæ Tractatus Quinque Theologici, Lyon.

Suárez, F., 1856-1878, Opera omnia, éd. C. Berton, Paris, L. Vivès, 28 vols.

Suárez, F., 1978-1991, Commentaria una cum quaestionibus in libros aristotelis 'De Anima', introd. et éd. S. Castellote Cubells, Madrid, Editorial Labor, 3 vols.

Schmaltz, T., 2008, Descartes on Causation, Oxford, Oxford University Press.

Schmid, S., 2015, «Efficient Causality: The Metaphysics of Production », in J. L. Fink, Suárez on Aristotelian Causality, Leyde, Brill, 84-120.

Schmutz, J., 2002, «Du péché de l'ange à la liberté d'indifférence. Les sources angélologiques de l'anthropologie moderne », Les études philosophiques 61 (2002/2), 169198.

Shields, C., 2012, "The Reality of Substantial Form: Suárez, Metaphysical Disputations XV », Daniel Schwartz (éd.), Interpreting Suárez : Critical Readings, Cambridge University Press, 39-61.

Shields, C., 2014, «Virtual Presence : Psychic Mereology in Francisco Suárez », in Partitioning the Soul. Debates from Plato to Leibniz, K. Corcilius et D. Perler (éds.), Berlin, De Gruyter, 199-218.

Spruit, L., 1995, Species intelligibilis. From Perception to Knowledge, vol. 2 Renaissance controversies, later scholasticism, and the elimination of the intelligible species in modern philosophy, Leyde, Brill.

Strawson, P.F., 1962, « Freedom and resentment », Proceedings of the British Academy 48, $1-25$.

Thomas d'Aquin, 1888-1906, Summa theologiae, in Opera omnia iussu impensaque Leonis XIII P. M. edita, vol. 4-12, Rome, Ex. Typographia Polyglotta S.C. de Propaganda Fide.

Van Inwagen, P., 2000, «Free Will Remains A Mystery », Philosophical Perspectives 14, $1-19$.

Zavalloni, R., 1951, Richard de Mediavilla et la controverse sur la pluralité des formes, Louvain 